\title{
Revegetação espontânea de voçoroca na região de Cerrado, Mato Grosso do Sul, Brasil
}

\author{
Adriana Guglieri-Caporal ${ }^{1,3}$, Francisco José Machado Caporal ${ }^{1}$, Arnildo Pott ${ }^{1}$, \\ Halisson Cesar Vinci-Carlos ${ }^{1}$ e Cedinara Arruda Santana Morales ${ }^{2}$
}

Recebido: 14.09.2010; aceito: 22.06.2011

\begin{abstract}
Spontaneous revegetation of gully in the Cerrado region, Mato Grosso do Sul State, Brazil). Gullies can be stabilized by restoration of original vegetation or another type of vegetation cover adapted to the new condition. This study aimed to characterize the spontaneous revegetation of a gully in cultivated pasture, isolated from cattle, in Mato Grosso do Sul State. We obtained data floristic and phytosociological and information related to the biology of the species involved of May/2009 to April/2010. We found 23 families, 49 genera and 71 species. Herbaceous-subshrubby (78.9\%), erect (63.4\%), hemicryptophyte (43.7\%) and autochoric species (60.0\%) predominated in the study area. The rainy period had $90 \%$ of the species in flower and 77.1\% in fruit. The attribute "bare soil" was high in terms of IV (51.6\%) and RC (72.5\%) in May/2009, values gradually reduced to $34.2 \%$ (IV) and $45.3 \%$ (RC) in February/2010. It is concluded that natural revegetation is under way. Such survey should be repeated within some years to monitore plant succession.
\end{abstract}

Key words: colonizing plants, recovery of degraded areas, soil erosion, Urochloa

RESUMO - (Revegetação espontânea de voçoroca na região de Cerrado, Mato Grosso do Sul, Brasil). As voçorocas podem ser estabilizadas pelo restabelecimento da vegetação original ou de outro tipo de cobertura vegetal adaptada à nova condição. Este estudo teve como objetivo caracterizar a revegetação natural de uma voçoroca localizada em área de pastagem cultivada, isolada do gado, em Mato Grosso do Sul. Foram obtidos dados florísticos e fitossociológicos de maio/2009 a abril/2010, e informações referentes à biologia das espécies envolvidas. Foram encontradas 23 famílias, 49 gêneros e 71 espécies. Predominaram as herbáceo-subarbustivas (78,9\%), eretas (63,4\%), hemicriptófitas (43,7\%) e autocóricas (60\%). O período chuvoso contou com $90 \%$ das espécies em floração e 77, 1\% em frutificação. O atributo "solo descoberto" apresentou elevado VI (51,6\%) e CR (72,50\%) em maio/2009, valores gradualmente reduzidos a 34,2\% (VI) e 45,3\% (CR) em fevereiro/2010. Conclui-se que a revegetação natural está em curso e sugere-se que o estudo seja repetido daqui a alguns anos, para monitorar a evolução da sucessão da vegetação.

Palavras-chave: erosão do solo, plantas colonizadoras, recuperação de áreas degradadas, Urochloa

\section{Introdução}

Práticas agropecuárias inadequadas podem desencadear processos erosivos que, associados a fatores edáficos e intempéries, resultam na formação de sulcos e ravinas e, em estágios mais avançados, voçorocas, as quais podem acarretar grandes perdas de solo, sérios prejuízos ambientais e a completa destruição da paisagem (Guerra 2001, Andrade et al. 2005, Rocha et al. 2005, Andres \& Werlang 2006). A formação de voçoroca se deve à passagem de água em um mesmo sulco repetidamente, por um longo período, o qual vai sendo ampliado pelo deslocamento de solo até dar origem a uma grande cavidade, em extensão e profundidade (Bahia et al. 1992). As voçorocas possuem profundidade maior que $0,5 \mathrm{~m}$ (Soil Science Society of America 2010), paredes laterais íngremes e, em geral, fundo chato, por onde flui a água durante os eventos chuvosos (Guerra 2001).

1. Universidade Federal de Mato Grosso do Sul, Centro de Ciências Biológicas e da Saúde, Laboratório de Botânica, Cidade Universitária, Caixa Postal 549, 79070-900 Campo Grande, MS, Brasil

2. Universidade Federal de Santa Maria, Programa de Pós-Graduação em Engenharia Florestal, Av. Roraima 1000, Prédio 44, Sala 5248, Cidade Universitária, 97105-900 Santa Maria, RS, Brasil

3. Autor para correspondência: adrianaguglieri@ig.com.br 
Voçorocamentos podem ser controlados por meio de práticas mecânicas e de revegetação. As primeiras referem-se a operações mecanizadas e/ou manuais para transporte de material, movimentação de terra, alocação e/ou remoção de rejeitos e construção de pequenas obras de contenção e dispositivos de drenagem superficial; a revegetação constitui-se no plantio de espécies adaptadas ao ambiente em questão, o que também é normalmente complementado com práticas de conservação de solo, como a incorporação de cobertura morta para proteção superficial e formação de serrapilheira (Andrade et al. 2005). Da mesma forma, reconhecido e contido o agente causador do processo, as voçorocas podem ser estabilizadas naturalmente por meio do restabelecimento da vegetação original ou de outra adaptada à nova condição. Este processo de regeneração natural é uma importante alternativa de recuperação de áreas degradadas que pode ter sucesso quando, além dos fatores ambientais, são conhecidas a disponibilidade de espécies locais e sua biologia, sobretudo os padrões de dispersão de sementes e frutos (Morretes 1992, Seitz 1994, Gandolfi \& Rodrigues 2007).

O presente trabalho teve como objetivo caracterizar a revegetação espontânea de uma voçoroca em pastagem cultivada com Urochloa brizantha (braquiarão, brizantão), respondendo as seguintes perguntas: a) quais as espécies envolvidas neste processo; b) qual o hábito, forma de crescimento, forma biológica e síndromes de dispersão mais comuns entre as espécies; c) quais os períodos de floração e frutificação das espécies; d) quais espécies destacaram-se em termos de frequência relativa, cobertura relativa e valor de importância na área durante o período estudado.

\section{Material e métodos}

Área de estudo - Fazenda Nova Esperança $\left(20^{\circ} 45^{\prime} 34,5^{\prime}\right.$ 'S e $\left.54^{\circ} 50^{\prime} 28,7^{\prime \prime} \mathrm{W}\right)$, com área aproximada de 4.000 ha, situada no município de Sidrolândia, a $37 \mathrm{~km}$ sul em linha reta de Campo Grande, Mato Grosso do Sul. O trabalho foi realizado em uma área de pastagem cultivada com Urochloa brizantha (braquiarão, brizantão) implantada em área originalmente de cerrado sensu stricto e cerradão na década de 1970. Esta invernada tem aproximadamente 100 ha e pastejo com diferimento como único manejo adotado. Em uso a lotação varia de 0,2 a 2 cabeças de gado bovino por hectare. A pastagem, no menor de seus lados, mantém contato com uma faixa de cerradão, fortemente impactado e desmatado.

$\mathrm{O}$ clima na região é do subtipo $A w$ tropical úmido de Köppen, temperatura média de $24^{\circ} \mathrm{C}$, precipitação média anual de $1.500 \mathrm{~mm}$, com estação chuvosa no verão (novembro a abril) e seca no inverno. De acordo com a Agraer (2010), os valores de precipitação mensal em Sidrolândia, no período de estudo, variaram de 70,6 (maio/2009) a $290 \mathrm{~mm}$ (janeiro/2010) (figura 1). O relevo é suave ondulado com predomínio de Latossolo Vermelho Distrófico (EMBRAPA 2008), a altitude varia de 513 a $526 \mathrm{~m}$ s.m.

Para o estudo, a voçoroca selecionada $\left(20^{\circ} 45^{\prime} 17,14^{\prime \prime} \mathrm{S}\right.$ e $\left.54^{\circ} 50^{\prime} 36,30^{\prime \prime} \mathrm{W}\right)$ foi previamente isolada por uma cerca com três fios de arame a fim de coibir o acesso do gado. Sua área total foi calculada com auxílio de GPS, sendo a extensão, largura e profundidade medidas com trena e a declividade obtida pela fórmula $\mathrm{dn} / \mathrm{dh} \times 100$, onde dn é a diferença de nível entre dois pontos e dh a distância horizontal entre esses dois pontos (Macedo et al. 2009). Em maio/2009, a voçoroca apresentava $3.327 \mathrm{~m}^{2}$ de área total, 130 m de extensão, 2,5 a 28,9 m de largura, 0,5 a $2,43 \mathrm{~m}$ de profundidade e declividade de $5,23 \%$ (figura 2 a-d).

Atributos físicos e químicos do solo - No topo da área há latossolo vermelho, enquanto na voçoroca afloram concreções de $\mathrm{Fe}$ e $\mathrm{Mn}$, e mais abaixo no perfil, basalto, limitando a profundidade da erosão. Foi realizada análise físico-química, a partir de uma amostra composta retirada de 12 pontos distribuídos

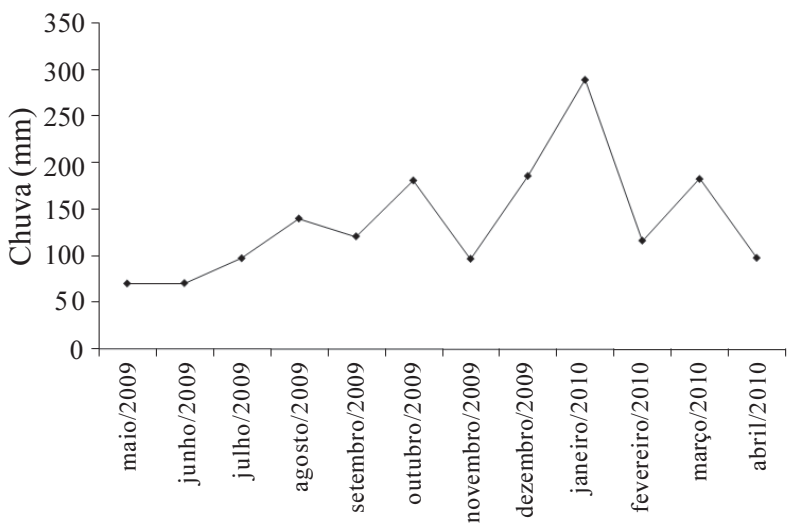

Figura 1. Precipitação mensal no município de Sidrolândia, MS, Brasil entre maio/2009 e abril/2010 (fonte: Agraer, 2010). Período seco: maio a setembro; período chuvoso: outubro a abril.

Figure 1. Monthly precipitation in city of Sidrolândia, MS, Brazil between May/2009 and April/2010 (source: Agraer, 2010). Dry season: May to September; rainy season: October to April. 
no centro e nas encostas da voçoroca. As análises foram feitas por Solos Laboratório de Análise, Consultoria e Informática Ltda., que segue a metodologia utilizada pela Embrapa Solos (Silva et al. 1998). Os resultados das análises (tabela 1) revelaram que o solo é argiloso, conforme a classificação textural de Lemos \& Santos (1996), e de pH fortemente ácido (Embrapa 2008). Verificaram-se teores considerados muito baixos para $\mathrm{P}$, médios para $\mathrm{Ca}$, altos para $\mathrm{Mg}$, $\mathrm{K}$ e $\mathrm{Ca}+\mathrm{Mg}$, e médios para matéria orgânica; em relação aos micronutrientes, verificaram-se teores de Fe inferiores aos limites críticos, altos para Mn, Zn e $\mathrm{Cu}$, e médios para B (Comissão de Fertilidade do Solo 1995, 2004).

Coleta de dados da vegetação - O acompanhamento do processo de revegetação natural da voçoroca foi realizado por meio de observações mensais de maio de 2009 a abril de 2010, e envolvendo um período seco (maio a setembro) e um chuvoso (outubro a abril).

1) Dados florísticos - mensalmente efetuou-se o levantamento florístico de toda as fanerógamas presentes na área de estudo, a partir de coletas de material botânico e observação das populações de plantas ocorrentes na voçoroca em estudo. Para identificação do material florístico foi utilizada bibliografia especializada, comparação com material de herbário e, quando necessária, consulta a especialistas. A identificação ao nível de famílias seguiu APG III (2009). O material coletado e identificado foi herborizado de acordo com as técnicas usuais e incorporado ao Herbário CGMS, da Universidade Federal de Mato Grosso do Sul campus Campo Grande.

Foi observada a localização dos indivíduos de cada espécie na voçoroca - encosta, centro e borda, tendo sido tratado como borda a porção de solo que se estende da margem da voçoroca até $2 \mathrm{~m}$ de distância.

Com base em observações em campo, cada espécie foi classificada quanto: ao hábito (erva, subarbusto, arbusto, árvore), conforme Pott et al. (2006), tendo sido tratada palmeira como um hábito à parte; à forma de crescimento (ascendente, decumbente, ereta, estolonífera, procumbente, rasteira, trepadeira, volúvel), de acordo com Radford et al. (1974); e à forma biológica (terófita, hemicriptófita, caméfita, geófita, fanerófita), conforme os grandes grupos do Sistema de Raunkier (1934), adaptado por Müller-Dombois \& Ellenberg (1974).

Foram incluídos apenas os nomes populares conhecidos no Mato Grosso do Sul, obtidos da literatura e dos moradores da região. A origem geográfica (exótica ou nativa do Brasil) foi baseada em bibliografia.

2) Dados referentes à fenologia e síndromes de dispersão - informações sobre os períodos de floração e frutificação das espécies foram obtidas a partir das observações mensais na área de estudo. Para a caracterização das síndromes de dispersão foram considerados o tamanho, a cor e a morfologia dos diásporos, incluindo a ornamentação das estruturas acessórias, classificando os frutos em autocóricos, anemocóricos e zoocóricos, conforme van der Pijl (1982).

3) Dados fitossociológicos - foram instaladas 56 parcelas permanentes de $0,25 \mathrm{~m}^{2}$ (figura 2e) dispostas ao longo da voçoroca que é orientada no sentido nortesul e, distribuídas nas encostas (37 parcelas) e no centro (19), em 12 linhas de 10 em 10 m no sentido leste-oeste.

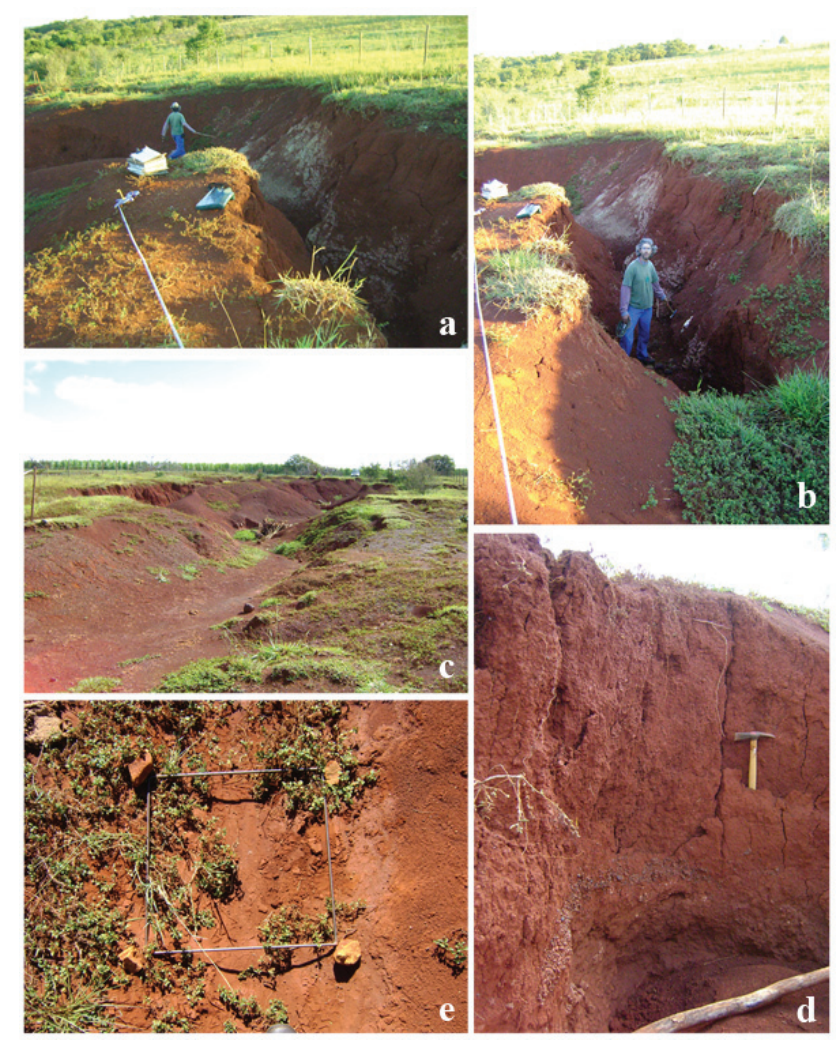

Figura 2. Voçoroca formada em pastagem cultivada com Urochloa brizantha, Fazenda Nova Esperança, Sidrolândia, MS, Brasil. a-c. Vista geral da voçoroca. d. Encosta da voçoroca. e. Parcela permanente no centro da voçoroca para amostragem da vegetação.

Figure 2. Gullies formed in pasture cultivated with Urochloa brizantha, Fazenda Nova Esperança, Sidrolândia, MS, Brazil. a-c. Overview of the gully. d. Slope of the gully. e. Permanent sample plots in the center of the gully for vegetation sampling. 
Tabela 1. Análise granulométrica e química do solo de uma voçoroca em pastagem cultivada com Urochloa brizantha após exclusão do gado, Fazenda Nova Esperança, Sidrolândia, MS, Brasil. pH - 1:2.5; $\mathrm{MO}-\mathrm{K}_{2} \mathrm{Cr}_{2} \mathrm{O}_{7}$; H - acetato de cálcio (pH 7); $\mathrm{P}$ e K - extrator de Mehlich I; Ca e Mg - EDTA; S - Soma das Bases; T - CTC; V - Sat de Bases; Fe-Mn-Zn-Cu - Mehlich -1; B - Água quente.

Table 1. Granulometric and chemistry analysis of soil of a gully in pasture cultivated with Urochloa brizantha after cattle exclusion, Fazenda Nova Esperança, Sidrolândia, MS, Brazil. pH - 1:2.5; $\mathrm{MO}-\mathrm{K}_{2} \mathrm{Cr}_{2} \mathrm{O}_{7} \mathrm{H}$ - calcium acetate (pH 7); P and K - extractors Mehlich I; Ca and Mg - EDTA; S - Soma of bases; T - CTC, V - Sat Bases; Fe-Mn-Zn-Cu - Mehlich -1; B - hot water.

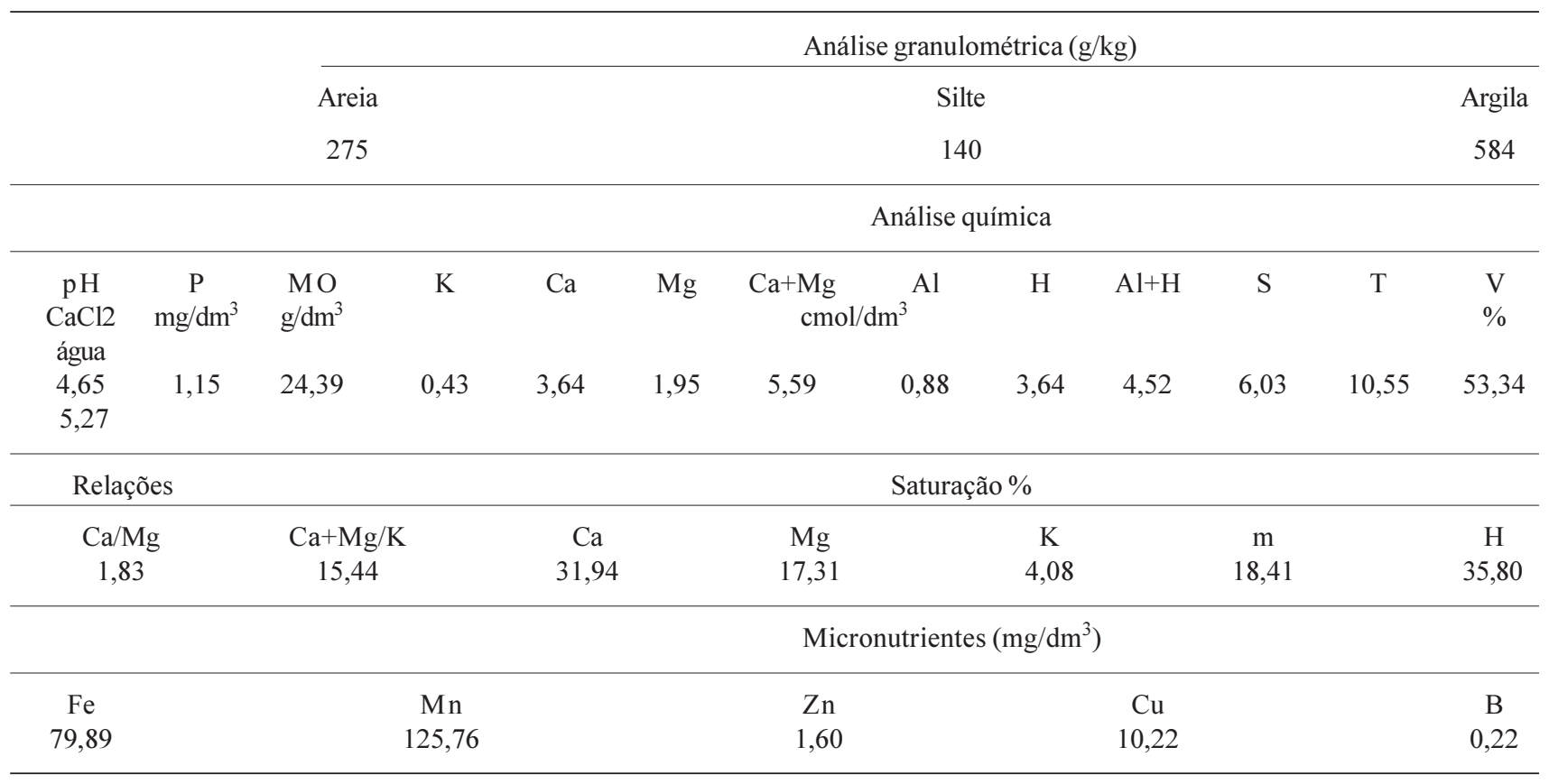

Os dados foram coletados em visitas trimestrais (maio, agosto, novembro/2009 e fevereiro/2010), tendo sido incluídas todas as espécies presentes nas unidades amostrais. Para avaliação visual da cobertura das espécies vegetais, bem como de solo descoberto, material seco (material vegetal morto e seco) e mantilho (material orgânico em decomposição), utilizou-se a escala de Daubenmire, segundo as interpretações de Müller-Dombois \& Ellenberg (1974).

Com base em dados de presença $\times$ ausência e na escala de cobertura, foram calculados os valores de frequência relativa (FR), cobertura relativa (CR) e valor de importância (VI) foram obtidos pelo programa PAST (Hammer et al. 2001).

\section{Resultados e Discussão}

$\mathrm{Na}$ área de estudo, foram registradas 23 famílias, 49 gêneros e 71 espécies de fanerógamas (tabela 2). As principais famílias em número de espécies foram Fabaceae (15 espécies), Poaceae (11), Asteraceae (nove) e Malvaceae (sete). Dentre as espécies pioneiras, as leguminosas (Fabaceae) têm-se destacado na revegetação de áreas degradadas, principalmente pela capacidade de associar-se a bactérias diazotróficas e poderem transportar $\mathrm{N}$ proveniente da fixação biológica do $\mathrm{N}_{2}$ atmosférico e nutrientes como $\mathrm{K}, \mathrm{Ca}$ e $\mathrm{Mg}$, das camadas mais profundas do solo à superfície (Oliveira et al. 2010).

Os gêneros mais representados na área foram Crotalaria, Digitaria, Solanum e Urochloa, com três espécies cada. Além de Urochloa brizantha, semeada como forrageira, outras seis espécies são exóticas e juntas representam cerca de $10 \%$ do total registrado.

Das espécies constatadas, 58 foram observadas na borda, 30 no centro e 23 na encosta da voçoroca. Aproximadamente 18\% (13 espécies) foram comuns às três zonas avaliadas, enquanto que $31(43,7 \%)$, nove $(12,7 \%)$ e duas $(2,8 \%)$ são exclusivas da borda, centro e encosta, respectivamente. Aparentemente os indivíduos que se desenvolvem na encosta e no centro da voçoroca atuam diretamente no processo de revegetação natural, como pioneiros e colonizadores, e aqueles da borda atuam na revegetação como fonte de sementes, frutos e propágulos vegetativos, além de contribuir na retenção e fixação de solo.

O componente herbáceo-subarbustivo contou com 56 espécies, o que corresponde a cerca de $80 \%$ 
Tabela 2. Espécies encontradas em uma voçoroca em pastagem cultivada com Urochloa brizantha após exclusão do gado, Fazenda Nova Esperança, Sidrolândia, MS, Brasil. * exótica; EN: encosta da voçoroca; CT: centro da voçoroca; BO: borda da voçoroca; HAB.: hábito; erv: erva; sub: subarbusto; arb: arbusto; arv: árvore; pal: palmeira; FC: forma de crescimento; asc: ascendente; dec: decumbente; ere: ereta; est: estolonífera; pro: procumbente; sue: subereta; ras: rasteira; FA: forma de vida; cam: caméfita; fan: fanerófita; geo: geófita; hem: hemicriptófita; ter: terófita; nome(s) popular(es); registro no Herbário CGMS; mat. veg.: material vegetativo.

Table 2. Species found in a gully in cultivated pasture with Urochloa brizantha after cattle exclusion, Fazenda Nova Esperança, Sidrolândia, MS, Brazil. * exotic; EN: slope of the gully; CT: center of the gully; BO: edge of the gully; HAB.: Habit; erv: herb, sub: subshrub; arb: shrub; arv: tree: pal: palm, FC: growth form; asc: ascending; dec: decumbent; ere: erect; est: stoloniferous; pro: procumbent; sue: suberect; ras: undergrowth; FA: life form; cam: chamaephytes; fan: phanerophytes; geo: geophytes; hem: hemicryptophytes; ter: therophytes; name(s) popular; record of CGMS Herbarium; mat. veg.: vegetative material.

\begin{tabular}{|c|c|c|c|c|c|c|c|c|}
\hline Família/Espécie & Nome(s) popular(es) & EN & $\mathrm{CT}$ & $\mathrm{BO}$ & HAB. & $\mathrm{FC}$ & FA & CGMS \\
\hline \multicolumn{9}{|l|}{ ANACARDIACEAE } \\
\hline Lithraea molleoides (Vell.) Engl. & aroeirinha-brava & - & - & $\mathrm{X}$ & arb & ere & fan & 29108 \\
\hline \multicolumn{9}{|l|}{ ANNONACEAE } \\
\hline Annona coriacea Mart. & araticum, ariticum & $X$ & $\mathrm{X}$ & $\mathrm{X}$ & arb/arv & ere & fan & 29090 \\
\hline Duguetia furfuracea (A. St.-Hil.) Saff. & ariticunzinho & $\mathrm{X}$ & - & $\mathrm{X}$ & arb & ere & fan & 29097 \\
\hline \multicolumn{9}{|l|}{ ARECACEAE } \\
\hline Acrocomia aculeata (Jacq.) Lodd. ex Mart. & bocaiúva & - & - & $\mathrm{X}$ & pal & ere & fan & mat.veg. \\
\hline \multicolumn{9}{|l|}{ ASTERACEAE } \\
\hline Acanthospermum australe (Loefl.) Kuntze & carrapicho-de-carneiro & $\mathrm{X}$ & $\mathrm{X}$ & $\mathrm{X}$ & erv & pro & ter & 25995 \\
\hline Bidens pilosa $\mathrm{L}$ & picão-preto & $\mathrm{X}$ & - & $\mathrm{X}$ & erv & ere & ter & 26012 \\
\hline Conyza canadensis (L.) Cronquist & buva & - & $\mathrm{X}$ & - & erv & ere & ter & 29092 \\
\hline Elephantopus angustifolius (Sw.) Gleason & língua-de-vaca & - & - & $\mathrm{X}$ & erv & ere & hem & 26255 \\
\hline Elephantopus mollis Kunth & fumo-bravo & $\mathrm{X}$ & - & - & erv & ere & hem & 26999 \\
\hline Pterocaulon lanatum Kuntze & branqueja & - & - & $\mathrm{X}$ & erv & ere & ter/hem & 25993 \\
\hline *Tridax procumbens L. & erva-de-touro & - & - & $X$ & erv & dec/asc & ter & 29082 \\
\hline Vernonia brasiliana (L.) Druce & assa-peixe & $\mathrm{X}$ & $X$ & $X$ & arb & ere & fan & 26988 \\
\hline Vernonia remotiflora Rich. & assa-peixe & - & - & $\mathrm{X}$ & sub & ere & fan & 26009 \\
\hline \multicolumn{9}{|l|}{ BORAGINACEAE } \\
\hline Euploca sp. & - & - & - & $\mathrm{X}$ & erv/sub & ere & hem & 29117 \\
\hline \multicolumn{9}{|l|}{ CARICACEAE } \\
\hline *Carica papaya L. & mamão & - & - & $\mathrm{X}$ & arv & ere & fan & mat.veg. \\
\hline \multicolumn{9}{|l|}{ COMMELINACEAE } \\
\hline *Commelina benghalensis L. & trapoeraba & - & $\mathrm{X}$ & - & erv & dec & hem & 26993 \\
\hline \multicolumn{9}{|l|}{ CONVOLVULACEAE } \\
\hline Evolvulus sp. & - & - & - & $\mathrm{X}$ & erv & asc & hem & 29087 \\
\hline Ipomoea grandifolia (Dammer) O’Donell & corda-de-viola & - & $\mathrm{X}$ & - & erv & vol & ter & 26010 \\
\hline \multicolumn{9}{|l|}{ CYPERACEAE } \\
\hline Cyperus cayennensis Willd. ex Link & tiririca & - & $\mathrm{X}$ & $\mathrm{X}$ & erv & ere & hem & 29114 \\
\hline \multicolumn{9}{|l|}{ EUPHORBIACEAE } \\
\hline Chamaesyce hyssopifolia (L.) Small & leiterinho & $X$ & - & $X$ & erv & ere/asc & ter & 25986 \\
\hline Chamaesyce prostrata (Aiton) Small & quebra-pedra & - & - & $\mathrm{X}$ & erv & pro & ter & 29083 \\
\hline Croton glandulosus $\mathrm{L}$. & gervão & $X$ & $\mathrm{X}$ & - & sub & ere & ter & 29088 \\
\hline Euphorbia serpens Kunth & quebra-pedra & - & - & $\mathrm{X}$ & erv & ras & ter & 29081 \\
\hline \multicolumn{9}{|l|}{ FABACEAE } \\
\hline Arachis oteroi Krapov. \& W.C. Gregory & amendoim-bravo & - & $\mathrm{X}$ & $\mathrm{X}$ & erv & ere/asc & geo & 29085 \\
\hline Chamaecrista nictitans (L.) Moench & - & - & - & $\mathrm{X}$ & $\mathrm{erv} / \mathrm{sub}$ & ere & hem & 29094 \\
\hline Chamaecrista serpens (L.) Greene & - & $\mathrm{X}$ & - & $\mathrm{X}$ & erv & asc/pro & hem & 29121 \\
\hline Crotalaria incana $\mathrm{L}$. & - & - & $\mathrm{X}$ & $\mathrm{X}$ & sub & ere & ter & 25994 \\
\hline Crotalaria lanceolata E. Mey. & - & - & - & $\mathrm{X}$ & erv & ere & hem & 29105 \\
\hline Crotalaria stipularia Desv. & - & $\mathrm{X}$ & - & $\mathrm{X}$ & $\mathrm{erv} / \mathrm{sub}$ & dec & hem & 26994 \\
\hline Desmodium barbatum (L.) Benth. & carrapicho & - & - & $\mathrm{X}$ & erv & asc/pro & hem & 29102 \\
\hline Desmodium incanum DC. & carrapicho & - & $\mathrm{X}$ & $\mathrm{X}$ & erv/sub & asc/pro & hem & 25987 \\
\hline Indigofera suffruticosa Mill. & anileira & - & $\mathrm{X}$ & - & arb & ere & fan & 26013 \\
\hline Mimosa polycarpa Kunth & aromita-preta & - & $\mathrm{X}$ & $\mathrm{X}$ & sub & ere & hem & 26002 \\
\hline Senna obtusifolia (L.) H.S. Irwin \& Barneby & fedegoso-branco & - & - & $\mathrm{X}$ & sub & ere & ter & 26985 \\
\hline Senna occidentalis (L.) Link & fedegoso & - & $\mathrm{X}$ & $\mathrm{X}$ & arb & ere & ter & 26011 \\
\hline
\end{tabular}


Tabela 2 (continuação)

\begin{tabular}{|c|c|c|c|c|c|c|c|c|}
\hline Família/Espécie & Nome(s) popular(es) & EN & $\mathrm{CT}$ & $\mathrm{BO}$ & HAB. & $\mathrm{FC}$ & FA & CGMS \\
\hline $\begin{array}{l}\text { Stylosanthes macrocephala M.B. Ferreira } \\
\quad \text { \& S. Costa }\end{array}$ & estilosantes & - & - & $X$ & sub & dec & hem & 26004 \\
\hline Stylosanthes scabra Vogel & estilosantes & $\mathrm{X}$ & $\mathrm{X}$ & $\mathrm{X}$ & sub & ere & hem & 26991 \\
\hline Zornia latifolia Sm. & - & $\mathrm{X}$ & - & - & erv & pro & hem & 25999 \\
\hline \multicolumn{9}{|l|}{ LAMIACEAE } \\
\hline $\begin{array}{l}\text { Hyptis suaveolens (L.) Poit. } \\
\text { MALPIGHIACEAE }\end{array}$ & tapera & - & $X$ & - & sub & ere & ter & 26986 \\
\hline $\begin{array}{l}\text { Heteropterys sp. } \\
\text { MALVACEAE }\end{array}$ & - & - & - & $X$ & arb & ere & fan & 26256 \\
\hline Ayenia tomentosa L. & - & - & - & $\mathrm{X}$ & erv/sub & ere & hem & 29118 \\
\hline Corchorus hirtus L. & - & $\mathrm{X}$ & - & $\mathrm{X}$ & erv & ere & ter & 26995 \\
\hline Sida linifolia Cav. & malvinha & $\mathrm{X}$ & - & $\mathrm{X}$ & erv & ere & ter & 26007 \\
\hline Sida rhombifolia L. & guanxuma & $\mathrm{X}$ & $\mathrm{X}$ & $\mathrm{X}$ & erv & ere & ter/hem & 25997 \\
\hline Sida urens L. & guanxuma & - & $\mathrm{X}$ & $\mathrm{X}$ & erv & pro/asc & ter & 26997 \\
\hline Sida sp. & malva & - & - & $\mathrm{X}$ & erv & ere & ter & 26257 \\
\hline Waltheria indica L. & malva-branca & $X$ & - & $X$ & erv/ sub & ere & hem & 25992 \\
\hline \multicolumn{9}{|l|}{ MORACEAE } \\
\hline $\begin{array}{l}\text { Maclura tinctoria (L.) D. Don ex Steud. } \\
\text { MYRSINACEAE }\end{array}$ & amora-brava & - & - & $X$ & arv & ere & fan & 29086 \\
\hline Myrsine guianensis (Aubl.) Kuntze & capororoca & - & - & $\mathrm{X}$ & arv & ere & fan & 29106 \\
\hline \multicolumn{9}{|l|}{ POACEAE } \\
\hline Digitaria bicornis (Lam.) Roem. \& Schult. & $\begin{array}{l}\text { capim-pé-de-galinha, } \\
\text { capim-de-capoeira }\end{array}$ & - & $\mathrm{X}$ & - & erv & dec & ter & 26008 \\
\hline Digitaria fuscescens (J. Presl.) Henrard & cuspo-do-taquarizano & - & - & $\mathrm{X}$ & erv & est & hem & 26992 \\
\hline Digitaria insularis (L.) Fedde & capim-amargoso & - & $\mathrm{X}$ & - & erv & ere & hem & 29084 \\
\hline *Hyparrhenia rufa (Nees) Stapf. & capim-jaraguá & $\mathrm{X}$ & $\mathrm{X}$ & $\mathrm{X}$ & erv & ere & hem & 25990 \\
\hline Paspalum notatum Flügge & $\begin{array}{l}\text { batatais, } \\
\text { capim-mato-grosso }\end{array}$ & $X$ & $X$ & $\mathrm{X}$ & erv & asc/pro & hem & 26000 \\
\hline Paspalum plicatulum Michx. & - & - & - & $\mathrm{X}$ & erv & ere & hem & 29100 \\
\hline Setaria parviflora (Poir.) Kerguélen & suçarana & $\mathrm{X}$ & $\mathrm{X}$ & $\mathrm{X}$ & erv & ere & ter/hem & 26001 \\
\hline Sporobolus indicus (L.) R. Br. & capim-touceirinha & - & - & $\mathrm{X}$ & erv & ere & hem & 26987 \\
\hline $\begin{array}{l}\text { *Urochloa brizantha (Hochst. ex } \\
\text { A. Rich.) Webster }\end{array}$ & braquiarão, brizantão & $X$ & $\mathrm{X}$ & $\mathrm{X}$ & erv & ere & hem & 26982 \\
\hline $\begin{array}{l}\text { *Urochloa humidicola (Rendle) Morrone } \\
\text { \& Zuloaga }\end{array}$ & humidícola & $X$ & $X$ & $X$ & erv & est & hem & 29098 \\
\hline $\begin{array}{l}\text { *Urochloa ruziziensis (R. Germ. \& } \\
\text { C.M. Evrard) Crins }\end{array}$ & braquiária & & & $\mathrm{X}$ & erv & dec & hem & 29115 \\
\hline \multicolumn{9}{|l|}{ POLYGALACEAE } \\
\hline Polygala extraaxillaris Chodat & - & - & - & $\mathrm{X}$ & erv & ere & hem & 29091 \\
\hline Polygala violacea Aubl. & - & - & - & $\mathrm{X}$ & erv & ere & hem & 29089 \\
\hline \multicolumn{9}{|l|}{ RHAMNACEAE } \\
\hline Rhamnidium elaeocarpum Reissek & cabrito & - & $\mathrm{X}$ & $\mathrm{X}$ & arv & ere & fan & 29107 \\
\hline \multicolumn{9}{|l|}{ RUBIACEAE } \\
\hline Diodia teres Walter & - & - & - & $\mathrm{X}$ & erv & asc & ter & 29093 \\
\hline Richardia brasiliensis B.A. Gomes & poaia & - & $\mathrm{X}$ & - & erv & pro & ter & 29103 \\
\hline Richardia stellaris (Cham. \& Schltdl.) Steud. & - & - & - & $\mathrm{X}$ & erv & asc/pro & hem & 26003 \\
\hline \multicolumn{9}{|l|}{ SMILACACEAE } \\
\hline Smilax cf. brasiliensis Spreng. & japecanga & - & - & $\mathrm{X}$ & sub & tre/asc & hem & 29120 \\
\hline \multicolumn{9}{|l|}{ SOLANACEAE } \\
\hline Solanum lycocarpum A. St.-Hil. & fruta-de-lobo, lobeira & - & - & $\mathrm{X}$ & arb/arv & ere & fan & 29101 \\
\hline Solanum paniculatum L. & jurubeba & $\mathrm{X}$ & $\mathrm{X}$ & - & arb & ere & cam & 25996 \\
\hline Solanum viarum Dunal & joá, juá-bravo & $\mathrm{X}$ & $\mathrm{X}$ & $\mathrm{X}$ & $\mathrm{erv} / \mathrm{sub}$ & ere/dec & ter/hem & 25998 \\
\hline \multicolumn{9}{|l|}{ VERBENACEAE } \\
\hline Lantana camara $\mathrm{L}$. & camará & - & $\mathrm{X}$ & - & arb & ere & cam/fan & 25989 \\
\hline
\end{tabular}


do total. Em geral, plantas herbáceas, por terem ciclos de vida relativamente curtos e altas taxas de reprodução, conseguem colonizar com extrema rapidez e dominar diferentes habitats (Junk \& Piedade 1997), desempenhando assim importante papel no processo de revegetação e na preparação do solo para desenvolvimento da vegetação lenhosa.

A maioria das espécies é ereta (cerca de 63,4\%), forma de crescimento observada entre as arbustivas e arbóreas, e em cerca de 53,6\% do total de herbáceas e subarbustivas. Em geral, plantas eretas criam condições de sombreamento para que outras se estabeleçam. Sementes de algumas espécies, por exemplo, não germinam sob pleno sol sendo favorecidas pelo leve sombreamento de gramíneas e arbustos (Regensburger 2004). As demais espécies do componente herbáceo-subarbustivo apresentaram-se como ascendentes, decumbentes, estoloníferas, procumbentes, rasteiras, trepadeiras e volúveis. Espécies estoloníferas desempenham importante papel na recuperação de áreas degradadas por promoverem a fixação e cobertura homogênea do solo e facilmente produzirem novos indivíduos por propagação vegetativa. Apenas as gramíneas Digitaria fuscescens e Urochloa humidicola são estoloníferas, entretanto, a primeira é pouco comum na área de estudo e a segunda, competidora agressiva com espécies nativas.

Hemicriptófita foi a forma de vida predominante (cerca de 43,7\%), seguida pela terófita (cerca de $28,2 \%)$, o que está relacionada à riqueza do componente herbáceo-subarbustivo do local (figura 3). Foi constatada uma única espécie geófita, Arachis oteroi. As geófitas podem apresentar tubérculos e as hemicriptófitas, rizomas e estolões, estruturas que as permitem sobreviver em períodos e condições adversos e contribuem na propagação vegetativa, atuando positivamente na revegetação natural. A estratégia das terófitas (anuais) para períodos e condições desfavoráveis é a rápida produção de um grande número de sementes. As anuais também contribuem na cobertura, acúmulo de matéria orgânica e cedem espaço a outras plantas, permitindo o estabelecimento e fixação de espécies perenes, secundárias e até terciárias.

Foi constatada a ocorrência de 53 espécies no período seco e 70 no chuvoso. Deste total, 17 espécies foram exclusivas do período chuvoso e uma do período seco, representada pela gramínea Digitaria fuscescens. No Brasil, esta espécie floresce o ano

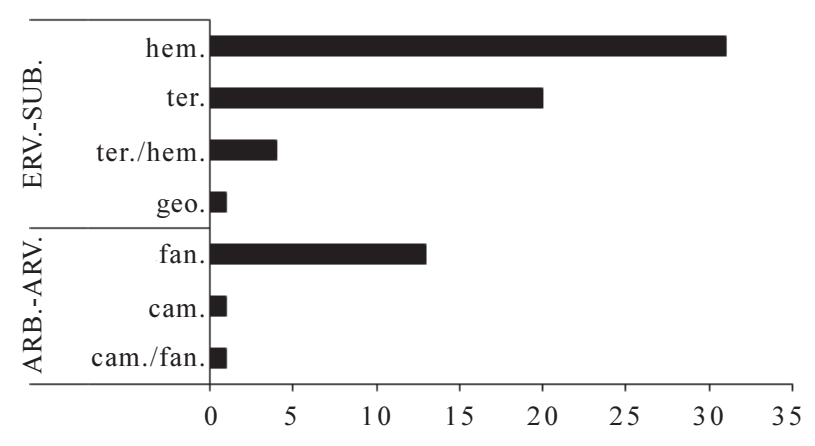

Figura 3. Número de espécies por forma de vida e componente vegetal em uma voçoroca em pastagem cultivada com Urochloa brizantha após exclusão do gado, Fazenda Nova Esperança, Sidrolândia, MS, Brasil. arb-arv: componente arbustivo-arbóreo; erv-sub: componente herbáceo-subarbustivo; cam.: caméfita; cam./fan.: caméfita a fanerófita; fan.: fanerófita; geo.: geófita; hem.: hemicriptófita; ter.: terófita; ter./he.: terófita a hemicriptófita.

Figure 3. Number of species by life form and plant component into a gully in cultivated pasture with Urochloa brizantha after cattle exclusion, Fazenda Nova Esperança, Sidrolândia, MS, Brazil. arb-arv: shrub-tree component; sub-erv: subshrub-herbaceous component; cam.: chamaephytes; cam/fan.: chamaephytes to the phanerophytes; fan.: phanerophytes; geo.: geophytes; hem.: hemicryptophytes; ter.: therophytes; ter/he.: therophytes to the hemicryptophytes.

inteiro, predominando de outubro a maio (CantoDorow 2001). Na área de estudo, foi observada uma pequena população na borda da voçoroca, que permaneceu estéril de maio a agosto de 2009, floresceu e frutificou em setembro e desapareceu depois, provavelmente suprimida pela competição com outras espécies, como Urochloa brizantha.

Do total de espécies amostradas, 11 permaneceram férteis, produzindo flores ou frutos, durante todo o período de estudo. Apenas cinco não foram encontradas férteis: Acrocomia aculeata, Carica papaya, Lithraea molleoides, Rhamnidium elaeocarpum e Smilax cf. brasiliensis, plantas de maior porte e ciclo mais longo do que as herbáceas, sendo todas ornitocóricas, indicando que essa forma de dispersão poderá aumentar a riqueza desse tipo de espécies. Convém mencionar que as duas primeiras espécies predominaram como plântulas, enquanto a última surgiu na área de estudo durante o último mês de coleta.

Constatou-se que a produção de flores e frutos se deu ao longo de todo o ano estudado (tabela 3 ). Durante o período seco, cerca de $66,04 \%$ das espécies avaliadas produziram flores e no chuvoso, $90 \%$. Neste período, a pluviosidade, o comprimento do dia, a temperatura e a umidade relativa médias mensais são 
Tabela 3. Período de floração e frutificação entre maio/2009 e abril/2010, e síndrome de dispersão das espécies encontradas em uma voçoroca em pastagem cultivada com Urochloa brizantha após exclusão do gado, Fazenda Nova Esperança, Sidrolândia, MS, Brasil. est: estéril; fl: florescido; fr: frutificado; jv: forma jovem; -: ocorrência não registrada; zoo: zoocoria; auto: autocoria; anemo: anemocoria.

Table 3. Period of flowering and fruiting between May/2009 and April/2010, and dispersal syndrome of species found in a gully in cultivated pasture with Urochloa brizantha after cattle exclusion, Fazenda Nova Esperança, Sidrolândia, MS, Brazil. est: sterile fl: flowered; fr: fruited; jv: young form; -: no occurrence unregistered; zoo: zoochory; auto: autocory; anemo: anemochory.

\begin{tabular}{|c|c|c|c|c|c|c|c|c|c|c|c|c|c|}
\hline \multirow[b]{2}{*}{ Espécie } & \multicolumn{6}{|c|}{ Período seco } & \multicolumn{6}{|c|}{ Período chuvoso } & \multirow{2}{*}{$\begin{array}{c}\text { Síndromes de } \\
\text { dispersão }\end{array}$} \\
\hline & maio & jun & jul & ago & set & out & nov & dez & jan & fev & mar & abr & \\
\hline Acanthospermum australe & fr & est & est & est & $\mathrm{fl}$ & $\mathrm{fl}, \mathrm{fr}$ & $\mathrm{fl}, \mathrm{fr}$ & $\mathrm{fl}, \mathrm{fr}$ & $\mathrm{fl}, \mathrm{fr}$ & $\mathrm{fl}, \mathrm{fr}$ & $\mathrm{fl}, \mathrm{fr}$ & $\mathrm{fl}, \mathrm{fr}$ & auto/zoo \\
\hline Acrocomia aculeata & - & - & - & - & - & - & - & - & - & - & jv & jv & zoo \\
\hline Annona coriacea & est & est & est & est & $\mathrm{fl}$ & fr & fr & fr & $\mathrm{fr}$ & fr & fr & fr & zoo \\
\hline Arachis oteroi & est & est & est & est & est & $\mathrm{fl}$ & $\mathrm{fl}$ & $\mathrm{fl}$ & $\mathrm{fl}, \mathrm{fr}$ & $\mathrm{fr}$ & $\mathrm{fr}$ & fr & auto \\
\hline Ayenia tomentosa & - & - & - & - & - & - & - & - & - & est & $\mathrm{fl}$ & fr & auto \\
\hline Bidens pilosa & $\mathrm{fr}$ & - & - & - & - & - & $\mathrm{fl}$ & $\mathrm{fl}, \mathrm{fr}$ & $\mathrm{fl}, \mathrm{fr}$ & $\mathrm{fr}$ & $\mathrm{fl}, \mathrm{fr}$ & - & zoo \\
\hline Carica papaya & $\mathrm{pl}$ & $\mathrm{pl}$ & $\mathrm{pl}$ & $\mathrm{pl}$ & $\mathrm{pl}$ & jv & jv & jv & jv & - & - & - & zoo \\
\hline Chamaecrista nictitans & - & - & - & - & - & est & $\mathrm{fl}$ & est & $\mathrm{fl}, \mathrm{fr}$ & - & - & - & auto \\
\hline Chamaecrista serpens & - & - & - & - & - & est & est & est & est & est & est & $\mathrm{fl}$ & auto \\
\hline Chamaesyce hyssopifolia & $\mathrm{fl}$ & $\mathrm{fl}$ & $\mathrm{fl}, \mathrm{fr}$ & $\mathrm{fl}, \mathrm{fr}$ & $\mathrm{fl}, \mathrm{fr}$ & $\mathrm{fl}, \mathrm{fr}$ & $\mathrm{fl}, \mathrm{fr}$ & $\mathrm{fl}, \mathrm{fr}$ & $\mathrm{fl}, \mathrm{fr}$ & $\mathrm{fl}$ & $\mathrm{fl}, \mathrm{fr}$ & $\mathrm{fl}, \mathrm{fr}$ & auto \\
\hline Chamaesyce prostrata & - & - & - & - & - & $\mathrm{fl}$ & $\mathrm{fl}, \mathrm{fr}$ & $\mathrm{fl}, \mathrm{fr}$ & $\mathrm{fl}, \mathrm{fr}$ & $\mathrm{fl}, \mathrm{fr}$ & - & - & auto \\
\hline Commelina benghalensis & - & - & - & est & $\mathrm{fl}$ & - & - & - & - & - & $\mathrm{fl}$ & - & auto \\
\hline Conyza canadensis & - & - & - & - & - & est & $\mathrm{fl}, \mathrm{fr}$ & $\mathrm{fl}, \mathrm{fr}$ & $\mathrm{fl}, \mathrm{fr}$ & $\mathrm{fl}, \mathrm{fr}$ & - & - & anemo \\
\hline Corchorus hirtus & - & - & - & $\mathrm{fl}$ & $\mathrm{fl}$ & $\mathrm{fl}$ & $\mathrm{fl}$ & $\mathrm{fl}$ & $\mathrm{fl}$ & $\mathrm{fl}, \mathrm{fr}$ & $\mathrm{fl}, \mathrm{fr}$ & $\mathrm{fl}, \mathrm{fr}$ & auto \\
\hline Crotalaria incana & $\mathrm{fl}, \mathrm{fr}$ & $\mathrm{fl}, \mathrm{fr}$ & $\mathrm{fl}, \mathrm{fr}$ & $\mathrm{fl}, \mathrm{fr}$ & $\mathrm{fl}, \mathrm{fr}$ & $\mathrm{fl}, \mathrm{fr}$ & fr & $\mathrm{fl}, \mathrm{fr}$ & $\mathrm{fr}$ & fr & $\mathrm{fl}, \mathrm{fr}$ & $\mathrm{fr}$ & auto \\
\hline Crotalaria lanceolata & - & - & - & - & - & - & - & - & est & $\mathrm{fl}, \mathrm{fr}$ & $\mathrm{fl}, \mathrm{fr}$ & $\mathrm{fl}, \mathrm{fr}$ & auto \\
\hline Crotalaria stipularia & est & est & est & est & $\mathrm{fl}$ & $\mathrm{fl}, \mathrm{fr}$ & $\mathrm{fl}, \mathrm{fr}$ & $\mathrm{fr}$ & $\mathrm{fl}, \mathrm{fr}$ & $\mathrm{fr}$ & est & est & auto \\
\hline Croton glandulosus & - & - & - & - & est & $\mathrm{fl}, \mathrm{fr}$ & $\mathrm{fl}, \mathrm{fr}$ & $\mathrm{fl}$ & $\mathrm{fl}, \mathrm{fr}$ & $\mathrm{fl}, \mathrm{fr}$ & $\mathrm{fl}, \mathrm{fr}$ & est & auto \\
\hline Cyperus cayennensis & - & - & - & - & - & est & $\mathrm{fl}, \mathrm{fr}$ & est & - & - & $\mathrm{fl}, \mathrm{fr}$ & $\mathrm{fl}, \mathrm{fr}$ & auto \\
\hline Desmodium barbatum & est & est & est & est & est & est & est & est & $\mathrm{fl}$ & est & est & est & zoo \\
\hline Desmodium incanum & $\mathrm{fl}$ & $\mathrm{fl}$ & $\mathrm{fl}$ & $\mathrm{fl}, \mathrm{fr}$ & $\mathrm{fl}, \mathrm{fr}$ & $\mathrm{fl}, \mathrm{fr}$ & $\mathrm{fl}$ & $\mathrm{fl}, \mathrm{fr}$ & $\mathrm{fl}, \mathrm{fr}$ & $\mathrm{fl}$ & $\mathrm{fl}, \mathrm{fr}$ & $\mathrm{fl}, \mathrm{fr}$ & zoo \\
\hline Digitaria bicornis & $\mathrm{fl}, \mathrm{fr}$ & $\mathrm{fl}, \mathrm{fr}$ & $\mathrm{fl}, \mathrm{fr}$ & $\mathrm{fl}, \mathrm{fr}$ & $\mathrm{fl}, \mathrm{fr}$ & $\mathrm{fl}, \mathrm{fr}$ & $\mathrm{fl}, \mathrm{fr}$ & $\mathrm{fl}, \mathrm{fr}$ & $\mathrm{fl}, \mathrm{fr}$ & $\mathrm{fl}, \mathrm{fr}$ & $\mathrm{fl}, \mathrm{fr}$ & $\mathrm{fl}, \mathrm{fr}$ & anemo \\
\hline Digitaria fuscescens & est & est & est & est & $\mathrm{fl}, \mathrm{fr}$ & - & - & - & - & - & - & - & auto \\
\hline Digitaria insularis & est & est & est & est & est & $\mathrm{fl}, \mathrm{fr}$ & $\mathrm{fl}, \mathrm{fr}$ & $\mathrm{fl}, \mathrm{fr}$ & $\mathrm{fl}, \mathrm{fr}$ & $\mathrm{fl}, \mathrm{fr}$ & est & est & anemo \\
\hline Diodiateres & - & - & - & - & - & est & $\mathrm{fl}$ & - & - & - & $\mathrm{fl}$ & - & auto \\
\hline Duguetia furfuracea & est & est & est & est & est & est & est & $\mathrm{fl}$ & $\mathrm{fl}$ & $\mathrm{fl}$ & $\mathrm{fl}, \mathrm{fr}$ & $\mathrm{fl}, \mathrm{fr}$ & zoo \\
\hline Elephantopus angustifolius & $\mathrm{fl}, \mathrm{fr}$ & $\mathrm{fl}, \mathrm{fr}$ & $\mathrm{fl}, \mathrm{fr}$ & $\mathrm{fl}, \mathrm{fr}$ & $\mathrm{fl}, \mathrm{fr}$ & $\mathrm{fl}$ & $\mathrm{fl}$ & $\mathrm{fl}$ & $\mathrm{fl}$ & $\mathrm{fl}, \mathrm{fr}$ & $\mathrm{fl}, \mathrm{fr}$ & $\mathrm{fl}, \mathrm{fr}$ & anemo \\
\hline Elephantopus mollis & est & est & $\mathrm{fl}$ & $\mathrm{fl}$ & $\mathrm{fl}, \mathrm{fr}$ & $\mathrm{fl}$ & $\mathrm{fl}$ & $\mathrm{fr}$ & - & - & - & $\mathrm{fl}, \mathrm{fr}$ & anemo \\
\hline Euphorbia serpens & - & - & - & - & - & $\mathrm{fl}, \mathrm{fr}$ & est & est & - & - & - & - & auto \\
\hline Euploca sp. & - & - & - & - & - & - & est & $\mathrm{fl}, \mathrm{fr}$ & est & est & est & $\mathrm{fl}$ & auto \\
\hline Evolvulus sp. & - & - & - & - & est & $\mathrm{fl}$ & - & - & - & - & - & - & auto \\
\hline Heteropterys sp. & $\mathrm{fl}$ & $\mathrm{fl}, \mathrm{fr}$ & fr & $\mathrm{fr}$ & fr & $\mathrm{fr}$ & est & est & est & est & est & est & anemo \\
\hline Hyparrhenia rufa & $\mathrm{fl}, \mathrm{fr}$ & $\mathrm{fl}, \mathrm{fr}$ & $\mathrm{fl}, \mathrm{fr}$ & $\mathrm{fl}, \mathrm{fr}$ & $\mathrm{fl}, \mathrm{fr}$ & $\mathrm{fl}, \mathrm{fr}$ & $\mathrm{fl}, \mathrm{fr}$ & $\mathrm{fl}, \mathrm{fr}$ & $\mathrm{fl}, \mathrm{fr}$ & $\mathrm{fl}, \mathrm{fr}$ & est & $\mathrm{fl}, \mathrm{fr}$ & $\begin{array}{c}\text { anemo, auto, } \\
\text { zoo }\end{array}$ \\
\hline Hyptis suaveolens & - & - & - & $\mathrm{fl}$ & $\mathrm{fl}$ & $\mathrm{fl}, \mathrm{fr}$ & - & - & - & - & $\mathrm{fl}$ & $\mathrm{fl}$ & auto \\
\hline Indigofera suffruticosa & $\mathrm{fl}$ & $\mathrm{fr}$ & $\mathrm{fr}$ & $\mathrm{fr}$ & $\mathrm{fl}, \mathrm{fr}$ & $\mathrm{fl}, \mathrm{fr}$ & $\mathrm{fr}$ & $\mathrm{fr}$ & - & - & - & - & auto \\
\hline Ipomoea grandifolia & $\mathrm{fl}$ & $\mathrm{fl}$ & $\mathrm{fl}$ & $\mathrm{fl}$ & $\mathrm{fl}$ & $\mathrm{fl}, \mathrm{fr}$ & $\mathrm{fl}$ & est & $\mathrm{fl}$ & $\mathrm{fl}$ & $\mathrm{fr}$ & $\mathrm{fr}$ & auto \\
\hline Lantana camara & $\mathrm{fr}$ & $\mathrm{fl}$ & $\mathrm{fl}$ & $\mathrm{fl}$ & $\mathrm{fl}$ & $\mathrm{fl}$ & $\mathrm{fl}, \mathrm{fr}$ & $\mathrm{fr}$ & $\mathrm{fl}, \mathrm{fr}$ & $\mathrm{fl}$ & $\mathrm{fl}, \mathrm{fr}$ & est & zoo \\
\hline Lithrea molleoides & est & est & est & est & est & est & est & est & est & est & est & est & zoo \\
\hline Maclura tinctoria & est & est & est & est & est & $\mathrm{fl}$ & est & est & est & est & est & est & zoo \\
\hline Mimosa polycarpa & $\mathrm{fr}$ & fr & fr & $\mathrm{fr}$ & $\mathrm{fr}$ & est & est & $\mathrm{fl}$ & $\mathrm{fl}$ & $\mathrm{fl}$ & $\mathrm{fl}, \mathrm{fr}$ & $\mathrm{fl}, \mathrm{fr}$ & auto \\
\hline Myrsine guianensis & est & est & est & est & est & est & est & est & $\mathrm{fl}$ & fr & est & est & zoo \\
\hline Paspalum notatum & $\mathrm{fl}, \mathrm{fr}$ & $\mathrm{fl}, \mathrm{fr}$ & $\mathrm{fl}, \mathrm{fr}$ & $\mathrm{fl}, \mathrm{fr}$ & est & est & $\mathrm{fl}, \mathrm{fr}$ & $\mathrm{fl}, \mathrm{fr}$ & $\mathrm{fl}, \mathrm{fr}$ & $\mathrm{fl}, \mathrm{fr}$ & $\mathrm{fl}, \mathrm{fr}$ & $\mathrm{fl}, \mathrm{fr}$ & auto \\
\hline Paspalum plicatulum & est & est & est & est & est & est & est & est & $\mathrm{fl}, \mathrm{fr}$ & $\mathrm{fl}, \mathrm{fr}$ & $\mathrm{fl}, \mathrm{fr}$ & est & auto \\
\hline Polygala extraaxilaris & - & - & - & - & - & $\mathrm{fl}$ & $\mathrm{fl}$ & $\mathrm{fl}$ & $\mathrm{fl}$ & $\mathrm{fl}$ & $\mathrm{fl}$ & $\mathrm{fl}, \mathrm{fr}$ & auto \\
\hline Polygala violacea & - & - & - & - & - & $\mathrm{fl}$ & - & - & $\mathrm{fl}$ & $\mathrm{fl}$ & - & - & auto \\
\hline Pterocaulon lanatum & fr & est & est & est & est & est & est & est & $\mathrm{fl}$ & $\mathrm{fl}$ & $\mathrm{fl}, \mathrm{fr}$ & $\mathrm{fl}, \mathrm{fr}$ & anemo \\
\hline Rhamnidium elaeocarpum & est & est & est & est & est & est & est & est & est & est & est & est & zoo \\
\hline
\end{tabular}




\begin{tabular}{|c|c|c|c|c|c|c|c|c|c|c|c|c|c|}
\hline \multirow[b]{2}{*}{ Espécie } & \multicolumn{6}{|c|}{ Período seco } & \multicolumn{6}{|c|}{ Período chuvoso } & \multirow{2}{*}{$\begin{array}{c}\text { Síndromes de } \\
\text { dispersão }\end{array}$} \\
\hline & maio & jun & jul & ago & set & out & nov & dez & jan & fev & mar & abr & \\
\hline Richardia brasiliensis & - & - & - & - & - & - & - & est & $\mathrm{fl}, \mathrm{fr}$ & - & $\mathrm{fl}$ & fr & auto \\
\hline Richardia stellaris & $\mathrm{fl}, \mathrm{fr}$ & $\mathrm{fl}, \mathrm{fr}$ & $\mathrm{fl}, \mathrm{fr}$ & $\mathrm{fl}, \mathrm{fr}$ & $\mathrm{fl}, \mathrm{fr}$ & $\mathrm{fr}$ & $\mathrm{fl}$ & $\mathrm{fl}, \mathrm{fr}$ & fr & $\mathrm{fl}$ & $\mathrm{fl}, \mathrm{fr}$ & $\mathrm{fl}, \mathrm{fr}$ & auto \\
\hline Senna obtusifolia & - & - & - & - & $\mathrm{fl}$ & $\mathrm{fl}, \mathrm{fr}$ & $\mathrm{fl}, \mathrm{fr}$ & $\mathrm{fl}, \mathrm{fr}$ & $\mathrm{fl}$ & $\mathrm{fl}, \mathrm{fr}$ & $\mathrm{fl}, \mathrm{fr}$ & $\mathrm{fl}, \mathrm{fr}$ & auto \\
\hline Senna occidentalis & $\mathrm{fr}$ & $\mathrm{fr}$ & $\mathrm{fr}$ & $\mathrm{fr}$ & fr & $\mathrm{fl}, \mathrm{fr}$ & $\mathrm{fl}, \mathrm{fr}$ & $\mathrm{fl}, \mathrm{fr}$ & $\mathrm{fl}, \mathrm{fr}$ & $\mathrm{fl}$ & $\mathrm{fl}, \mathrm{fr}$ & fr & auto \\
\hline Setaria parviflora & $\mathrm{fl}, \mathrm{fr}$ & $\mathrm{fl}, \mathrm{fr}$ & $\mathrm{fl}, \mathrm{fr}$ & $\mathrm{fl}, \mathrm{fr}$ & $\mathrm{fl}, \mathrm{fr}$ & $\mathrm{fl}, \mathrm{fr}$ & $\mathrm{fl}, \mathrm{fr}$ & $\mathrm{fl}, \mathrm{fr}$ & $\mathrm{fl}, \mathrm{fr}$ & $\mathrm{fl}, \mathrm{fr}$ & $\mathrm{fl}, \mathrm{fr}$ & $\mathrm{fl}, \mathrm{fr}$ & auto \\
\hline Sida linifolia & est & est & est & $\mathrm{fl}$ & $\mathrm{fl}$ & $\mathrm{fl}$ & $\mathrm{fl}, \mathrm{fr}$ & $\mathrm{fl}$ & $\mathrm{fl}, \mathrm{fr}$ & $\mathrm{fl}, \mathrm{fr}$ & fr & $\mathrm{fl}, \mathrm{fr}$ & auto \\
\hline Sida rhombifolia & fr & est & est & $\mathrm{fl}$ & $\mathrm{fl}$ & $\mathrm{fl}$ & $\mathrm{fl}, \mathrm{fr}$ & $\mathrm{fl}, \mathrm{fr}$ & $\mathrm{fl}, \mathrm{fr}$ & $\mathrm{fl}, \mathrm{fr}$ & $\mathrm{fl}, \mathrm{fr}$ & $\mathrm{fl}, \mathrm{fr}$ & auto \\
\hline Sida urens & - & - & est & $\mathrm{fl}$ & $\mathrm{fl}$ & $\mathrm{fl}$ & est & est & est & $\mathrm{fl}$ & $\mathrm{fl}$ & $\mathrm{fl}, \mathrm{fr}$ & auto \\
\hline Sida $\mathrm{sp}$ & $\mathrm{fl}$ & $\mathrm{fl}$ & $\mathrm{fl}$ & $\mathrm{fr}$ & $\mathrm{fr}$ & - & - & - & $\mathrm{fl}$ & - & - & - & auto \\
\hline Smilax cf. brasiliensis & - & - & - & - & - & - & - & - & - & - & - & est & zoo \\
\hline Solanum lycocarpum & est & est & est & est & est & est & est & est & $\mathrm{fl}, \mathrm{fr}$ & $\mathrm{fl}, \mathrm{fr}$ & $\mathrm{fr}$ & $\mathrm{fr}$ & zoo \\
\hline Solanum paniculatum & $\mathrm{fl}$ & $\mathrm{fl}$ & $\mathrm{fl}$ & $\mathrm{fl}$ & $\mathrm{fl}$ & $\mathrm{fl}, \mathrm{fr}$ & est & $\mathrm{fl}$ & $\mathrm{fl}, \mathrm{fr}$ & $\mathrm{fl}$ & $\mathrm{fl}, \mathrm{fr}$ & $\mathrm{fl}$ & zoo \\
\hline Solanum viarum & $\mathrm{fl}, \mathrm{fr}$ & $\mathrm{fl}, \mathrm{fr}$ & $\mathrm{fl}, \mathrm{fr}$ & $\mathrm{fl}, \mathrm{fr}$ & $\mathrm{fl}, \mathrm{fr}$ & $\mathrm{fl}, \mathrm{fr}$ & $\mathrm{fl}, \mathrm{fr}$ & $\mathrm{fl}$ & $\mathrm{fr}$ & $\mathrm{fl}, \mathrm{fr}$ & $\mathrm{fr}$ & $\mathrm{fr}$ & zoo \\
\hline Sporobolus indicus & est & est & est & $\mathrm{fl}, \mathrm{fr}$ & $\mathrm{fl}, \mathrm{fr}$ & $\mathrm{fl}, \mathrm{fr}$ & $\mathrm{fl}, \mathrm{fr}$ & $\mathrm{fl}, \mathrm{fr}$ & $\mathrm{fl}, \mathrm{fr}$ & est & est & est & auto \\
\hline Stylosanthes macrocephala & est & est & est & est & est & est & est & est & est & est & est & $\mathrm{fl}$ & auto \\
\hline Stylosanthes scabra & $\mathrm{fl}$ & $\mathrm{fl}$ & $\mathrm{fl}$ & $\mathrm{fl}$ & $\mathrm{fl}$ & $\mathrm{fl}$ & $\mathrm{fl}$ & $\mathrm{fl}$ & est & $\mathrm{fl}$ & $\mathrm{fl}$ & $\mathrm{fl}$ & auto \\
\hline Tridax procumbens & - & - & - & - & - & $\mathrm{fl}$ & $\mathrm{fl}$ & - & - & - & - & - & anemo \\
\hline Urochloa brizantha & $\mathrm{fl}, \mathrm{fr}$ & $\mathrm{fl}, \mathrm{fr}$ & $\mathrm{fl}, \mathrm{fr}$ & $\mathrm{fl}, \mathrm{fr}$ & $\mathrm{fl}, \mathrm{fr}$ & $\mathrm{fl}, \mathrm{fr}$ & $\mathrm{fl}, \mathrm{fr}$ & $\mathrm{fl}, \mathrm{fr}$ & $\mathrm{fl}, \mathrm{fr}$ & $\mathrm{fl}, \mathrm{fr}$ & $\mathrm{fl}, \mathrm{fr}$ & $\mathrm{fl}, \mathrm{fr}$ & auto \\
\hline Urochloa humidicola & - & - & - & est & est & est & est & $\mathrm{fl}, \mathrm{fr}$ & $\mathrm{fl}, \mathrm{fr}$ & $\mathrm{fl}, \mathrm{fr}$ & $\mathrm{fl}, \mathrm{fr}$ & $\mathrm{fl}, \mathrm{fr}$ & auto \\
\hline Urochloa ruziziensis & - & - & - & - & - & - & - & - & est & est & $\mathrm{fl}, \mathrm{fr}$ & $\mathrm{fl}, \mathrm{fr}$ & auto \\
\hline Vernonia brasiliana & $\mathrm{fl}$ & $\mathrm{fl}$ & $\mathrm{fl}$ & $\mathrm{fl}$ & $\mathrm{fl}$ & $\mathrm{fl}$ & $\mathrm{fl}$ & $\mathrm{fl}, \mathrm{fr}$ & $\mathrm{fl}, \mathrm{fr}$ & est & $\mathrm{fl}$ & $\mathrm{fl}$ & anemo \\
\hline Vernonia remotiflora & $\mathrm{fl}$ & $\mathrm{fl}$ & $\mathrm{fl}$ & $\mathrm{fl}$ & $\mathrm{fl}$ & $\mathrm{fl}$ & $\mathrm{fl}$ & $\mathrm{fl}, \mathrm{fr}$ & $\mathrm{fl}, \mathrm{fr}$ & est & $\mathrm{fl}$ & $\mathrm{fl}$ & anemo \\
\hline Waltheria indica & $\mathrm{fl}$ & $\mathrm{fl}$ & $\mathrm{fl}$ & $\mathrm{fl}$ & $\mathrm{fl}$ & $\mathrm{fl}$ & $\mathrm{fl}$ & $\mathrm{fl}, \mathrm{fr}$ & $\mathrm{fl}$ & $\mathrm{fl}$ & $\mathrm{fl}, \mathrm{fr}$ & $\mathrm{fl}, \mathrm{fr}$ & auto \\
\hline Zornia latifolia & $\mathrm{fl}$ & $\mathrm{fl}$ & $\mathrm{fl}$ & $\mathrm{fl}$ & $\mathrm{fl}$ & $\mathrm{fl}$ & $\mathrm{fl}, \mathrm{fr}$ & est & est & est & $\mathrm{fl}$ & $\mathrm{fl}, \mathrm{fr}$ & auto/zoo \\
\hline
\end{tabular}

maiores e propiciam o aumento da floração (Batalha et al. 1997). Como consequência, a produção de frutos foi proporcionalmente maior no período chuvoso (cerca de $77,1 \%$ das espécies), em contraste com o seco (cerca de 45,3\%).

Os componentes herbáceo-arbustivo e arbustivo-arbóreo apresentaram padrões de floração e frutificação distintos (figura 4). Observou-se que no primeiro, o maior percentual de espécies em floração ocorreu em novembro e janeiro (cerca de $76 \%$ cada) e o de frutificação, em março $(60 \%)$ e abril (cerca de 70\%); no segundo, os maiores percentuais de floração foram atingidos em outubro (cerca de $43 \%$ ) e janeiro (cerca de $54 \%$ ), e os de frutificação, em janeiro e março (cerca de $46 \%$ cada). Tais resultados são similares a outros estudos realizados no Cerrado (Mantovani \& Martins 1988, Batalha et al. 1997, Batalha \& Mantovani 2000), que mostraram que as espécies herbáceas e subarbustivas florescem entre os meses de janeiro e março e frutificam ao final do período chuvoso, em março e abril, enquanto que as arbustivas e arbóreas florescem de setembro a novembro e frutificam ao longo do período chuvoso.
A síndrome de dispersão mais frequente foi a autocoria (42 espécies, 60\%) (tabela 3); nesta síndrome, os frutos e sementes caem próximo da planta-mãe colaborando com o restabelecimento da vegetação local. A zoocoria foi representada por 15 espécies (cerca de $21,4 \%$ ), enquanto que a anemocoria por 10 (cerca de 14,3\%). De acordo com Seitz (1994), em casos extremos de degradação da vegetação, onde esta é praticamente ausente, as espécies anemocóricas são as primeiras a se estabelecer. Cabe salientar que em síndromes como anemocoria e zoocoria, onde estão envolvidos agentes dispersores como o vento e os animais, respectivamente, a planta-mãe pode se encontrar a maiores distâncias do local de estabelecimento do fruto ou semente. Neste caso, a voçoroca estudada não depende essencialmente das espécies zoocóricas e anemocórias in situ e pode ser favorecida pelos remanescentes de cerradão e cerrado sensu stricto próximos; por outro lado, pode-se supor que as anemocóricas sejam prejudicadas pela plantação de eucalipto próximo à área de estudo, que tende a barrar o vento vindo principalmente do noroeste.

A gramínea Hyparrhenia rufa pode eventualmente apresentar os três mecanismos de dispersão. 


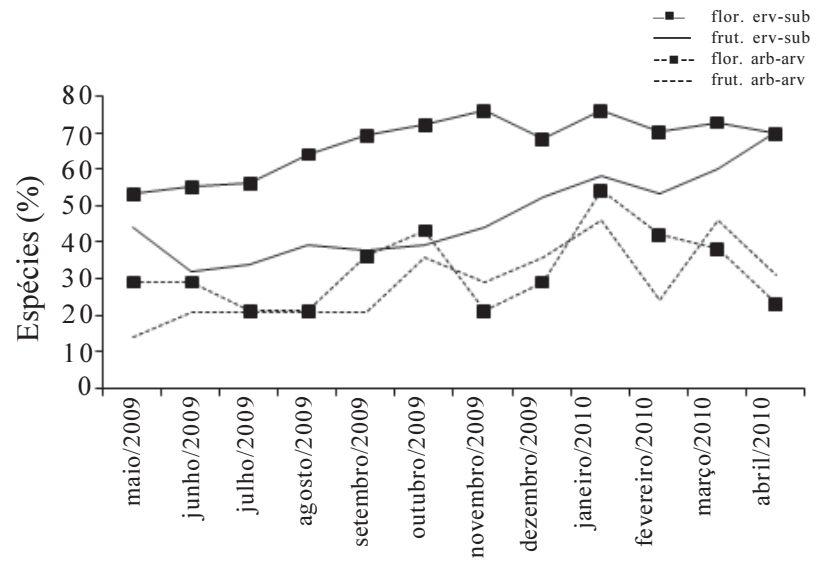

Figura 4. Percentual de espécies em floração e frutificação por componente vegetal entre maio/2009 e abril/2010 em uma voçoroca em pastagem cultivada com Urochloa brizantha após exclusão do gado, Fazenda Nova Esperança, Sidrolândia, MS, Brasil. arb-arv: componente arbustivo-arbóreo; erv-sub: componente herbáceosubarbustivo; flor.: floração; frut.: frutificação. Período seco: maio a setembro; período chuvoso: outubro a abril.

Figure 4. Percentage of species flowering and fruiting by plant component between May/2009 and April/2010 in a gully in cultivated pasture with Urochloa brizantha after cattle exclusion, Fazenda Nova Esperança, Sidrolândia, MS, Brazil. arb-arv: shrubtree component; sub-erv: subshrub-herbaceous component; flower: flowering; frut.: fruiting. Dry season: May to September; rainy season: October to April.

As cariopses são amplas e leves sendo facilmente disseminadas pelo vento e as espiguetas com longas aristas podem ser carregadas por animais. Dissemínulos de várias espécies podem ainda ter dispersão hidrocórica secundária, principalmente os que caem na encosta.

O maior percentual de espécies autocóricas, anemocóricas e zoocóricas em frutificação ocorreram no período chuvoso (figura 5). Apesar de uma queda na produção de frutos das espécies anemocóricas em outubro, início da estação chuvosa, os maiores percentuais (cerca de $56 \%$ a $70 \%$ ) foram observados entre dezembro e fevereiro e em abril. Weiser \& Godoy (2001) apontaram a predominância da frutificação de espécies anemocóricas no período seco e de zoocóricas e autocóricas, no início e final do período chuvoso, respectivamente. Entretanto, a maioria dos estudos fenológicos realizados no Cerrado (Mantovani \& Martins 1988, Batalha et al. 1997, Batalha \& Mantovani 2000) indicaram que a frutificação das espécies autocóricas e anemocóricas é mais comum no período seco, enquanto que a das zoocóricas ocorre principalmente durante o período chuvoso. No período seco, a dispersão dos frutos anemocóricos é favorecida pelos ventos que ocorrem com maior força e frequência. Além disso, nesse período, o pericarpo dos frutos autocóricos e anemocóricos são desidratados, o que provoca a sua deiscência (Mantovani \& Martins 1988). Modificações nas condições ambientais podem alterar padrões reprodutivos esperados (Antunes \& Ribeiro 1999), o que pode explicar os resultados obtidos pelo presente estudo.

No componente arbustivo-arbóreo predominou a zoocoria (cerca de 71,4\%), enquanto que no herbáceosubarbustivo predominou a autocoria (cerca de 71,4\%) seguida pela anemocoria (cerca de 14,3\%) (figura 6). Estes resultados estão de acordo com Mantovani \& Martins (1988), Batalha et al. (1997), Batalha \& Mantovani (2000) e Weiser \& Godoy (2001), ratificando que em áreas de Cerrado a zoocoria está melhor representada entre as espécies arbustivo-arbóreas, e autocoria e anemocoria, entre as herbáceosubarbustivas.

Observou-se a ocorrência de várias espécies com conhecido potencial para recuperação de áreas degradadas, entre elas, Annona coriacea, Duguetia furfuracea, Maclura tinctoria, Mimosa polycarpa, Rhamnidium elaeocarpum e Solanum lycocarpum, a primeira já recomendada para recuperação de áreas com voçorocas (Pott et al. 2006). Isto nos permite inferir que as mesmas contribuirão com o sucesso da

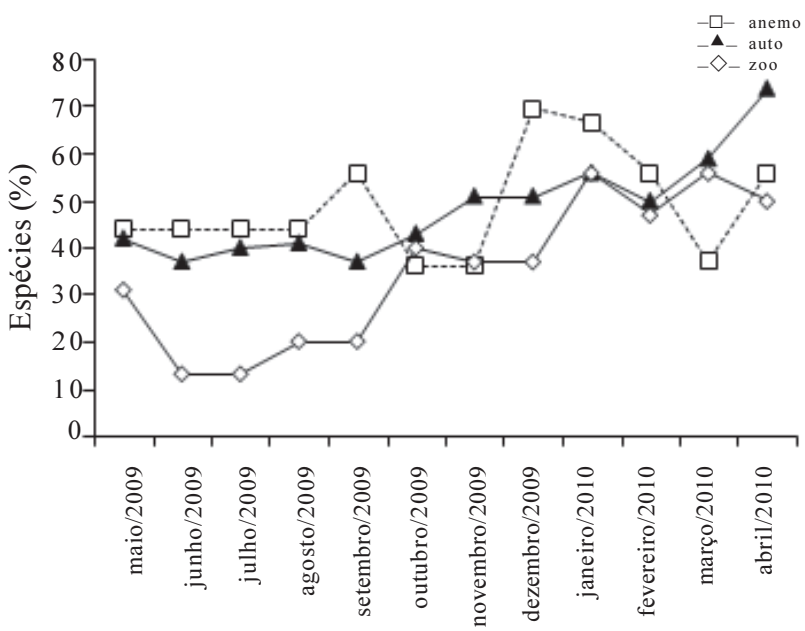

Figura 5. Percentual de espécies em frutificação por síndrome de dispersão entre maio/2009 a abril/2010 em uma voçoroca em pastagem cultivada com Urochloa brizantha após exclusão do gado, na Fazenda Nova Esperança, Sidrolândia, MS, Brasil. anemo: anemocoria; auto: autocoria; zoo: zoocoria. Período seco: maio a setembro; período chuvoso: outubro a abril.

Figure 5. Percentage of fruiting species by dispersal syndrome between May/2009 April/2010 in a gully in pasture cultivated with Urochloa brizantha after cattle exclusion, Fazenda Nova Esperança, Sidrolândia, MS, Brazil. anemo: anemochory; auto: autocory; zoo: zoochory. Dry season: May to September; rainy season: October to April. 


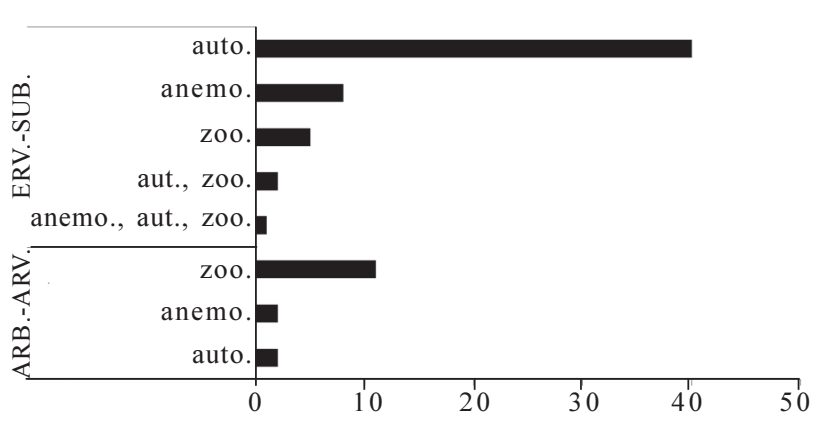

Figura 6. Número de espécies por síndrome de dispersão e componente vegetal em uma voçoroca em pastagem cultivada com Urochloa brizantha após exclusão do gado, Fazenda Nova Esperança, Sidrolândia, MS, Brasil. anemo: anemocoria; auto: autocoria; zoo: zoocoria.

Figure 6. Number of species by dispersal syndrome and plant compound into a gully in cultivated pasture with Urochloa brizantha after cattle exclusion, Fazenda Nova Esperança, Sidrolândia, MS, Brazil. anemo: anemochory; auto: autocory; zoo: zoochory.

revegetação natural da voçoroca.

Através da estabilização da curva espécies-área (Braun-Blanquet 1979) e da determinação da área mínima (Matteucci \& Colma 1982), a suficiência amostral foi confirmada. Os cálculos realizados (tabela 4) mostraram que o "solo descoberto" foi elevado em termos de VI e CR. Em maio/2009 (período seco), este atributo apresentou 51,6\% e 72,5\% para VI e CR, respectivamente, valores gradualmente reduzidos a $34,2 \%(\mathrm{VI})$ e $45,3 \%$ (CR) em fevereiro/2010 (período chuvoso). Esta redução está relacionada apenas em parte à sazonalidade e tende a ser gradual e efetiva na área de estudo, uma vez que o processo de revegetação natural está em curso.

$\mathrm{O}$ aumento na cobertura do solo está relacionado, em parte, ao aumento de CR dos atributos "material morto" e "mantilho", que variaram de 6,1\% e 3,0\% (maio/2009) a 9,4\% e 7,7\% (fevereiro/2010), respectivamente. $\mathrm{O}$ acúmulo desses componentes é importante na ciclagem de nutrientes e proteção do solo contra as intempéries e consequente erosão. Também contribuíram com o aumento de cobertura do solo ervas, subarbustos e arbustos que se desenvolveram no local e que somaram CR entre 18,5\% (maio/2009) e 37,4\% (fevereiro/2010).

Acanthospermum australe, Paspalum notatum e Urochloa brizantha obtiveram altos valores para CR e FR durante o período de estudo. Se por um lado, essas espécies promovem considerável cobertura do solo, por outro, seu caráter competidor agressivo (Kissmann 1997, Kissmann \& Groth 1999), pode interferir no estabelecimento e desenvolvimento de outras espécies vegetais, diminuindo assim a riqueza florística local que é importante nos processos de recuperação de áreas degradadas.

Foi observado para $A$. australe redução do VI entre novembro/2009 (17,3\%) e fevereiro/2010 $(14,7 \%)$. É provável que esta redução esteja relacionada ao aumento de CR de espécies de maior porte como U. brizantha, Mimosa polycarpa e Senna spp., por promoverem sombreamento. Acanthospermum australe é anual, procumbente e heliófila, necessitando de iluminação abundante para o seu desenvolvimento, senão seu crescimento vegetativo e frutificação são prejudicados (Kissmann \& Groth 1999). Assim, os processos naturais de revegetação e sucessão ecológica podem controlar a sua ocorrência.

Paspalum notatum apresentou aumento gradual de CR, inclusive no período seco $(3,7 \%$ a $6,8 \%)$, quando poucas espécies apresentaram CR acima de $1 \%$. Espécie procumbente-ascendente, perene, com vigorosos rizomas horizontais de entrenós curtos, forma uma densa cobertura sobre o solo (Kismann 1997), conseguindo manter-se em condições de baixa fertilidade e pouca profundidade do solo, variações de umidade e competição com outras espécies vegetais (Pedreira \& Pedreira 2006), incluindo Urochloa spp., todos fatores positivos no caso da voçoroca estudada.

Os altos valores de CR e FR de $U$. brizantha indicam que a sucessão poderá ser mais lenta na área, uma vez que plantas desta espécie produzem compostos alelopáticos capazes de afetar a germinação (Souza Filho et al. 1997) e, consequentemente, a frequência de outras espécies locais.

Urochloa humidicola em maio/2009 (período seco) apresentou baixos valores para CR $(0,24 \%)$ e FR $(0,55 \%)$, mas no fim do ano de estudo (período chuvoso) apareceu com $4,1 \%$ e $4,6 \%$, respectivamente CR e FR. Por ocorrer preferencialmente em solo úmido é provável que a pluviosidade tenha colaborado com sua rápida expansão na área. Além de seu potencial alelopático (Souza Filho et al. 1997) esta espécie se propaga facilmente de modo vegetativo, com formação de novos colmos a partir de estolões e rizomas (Kissmann 1997, Lorenzi 2000), sendo também útil na recuperação de voçorocas, embora possa retardar a sucessão, assim como U. brizantha.

Ao longo do ano estudado a CR do "solo descoberto" variou de $75,4 \%$ a $42,0 \%$ no centro e de 
Tabela 4. Dados fitossociológicos de atributos e espécies em quatro épocas, em uma voçoroca em pastagem cultivada com Urochloa brizantha após exclusão do gado, Fazenda Nova Esperança, Sidrolândia, MS, Brasil. VI: Valor de Importância; CA: Cobertura Absoluta; FA: Frequência Absoluta; CR: Cobertura Relativa; FR: Frequência Relativa.

Table 4. Phytosociological data of attributes and species in four seasons in a gully in cultivated pasture with Urochloa brizantha after cattle exclusion, Fazenda Nova Esperança, Sidrolândia, MS, Brazil. VI: Importance Value; CA: Absolute Coverage; FA: Absolute Frequency, CR: Relative Coverage; FR: Relative Frequency.

\begin{tabular}{|c|c|c|c|c|c|}
\hline maio/2009 & $\begin{array}{l}\text { CA } \\
(\%)\end{array}$ & $\begin{array}{l}\text { FA } \\
(\%)\end{array}$ & $\begin{array}{l}\text { CR } \\
(\%)\end{array}$ & $\begin{array}{l}\text { FR } \\
(\%)\end{array}$ & $\begin{array}{l}\text { VI } \\
(\%)\end{array}$ \\
\hline solo descoberto & $4.600,0$ & 100,0 & 72,5 & 30,8 & 51,6 \\
\hline Acanthospermum australe & 582,5 & 51,8 & 9,2 & 15,9 & 12,5 \\
\hline material morto & 385,0 & 55,4 & 6,1 & 17,0 & 11,5 \\
\hline mantilho & 190,0 & 46,4 & 3,0 & 14,3 & 8,6 \\
\hline Paspalum notatum & 235,0 & 28,6 & 3,7 & 8,8 & 6,3 \\
\hline Urochloa brizantha & 145,0 & 8,9 & 2,3 & 2,8 & 2,5 \\
\hline Sida rhombifolia & 65,0 & 10,7 & 1,0 & 3,3 & 2,2 \\
\hline Mimosa polycarpa & 40,0 & 3,5 & 0,6 & 1,1 & 0,9 \\
\hline Digitaria bicornis & 30,0 & 3,5 & 0,5 & 1,1 & 0,8 \\
\hline Arachis oteroi & 15,0 & 1,8 & 0,2 & 0,6 & 0,4 \\
\hline Sida linifolia & 15,0 & 1,8 & 0,2 & 0,6 & 0,4 \\
\hline Urochloa humidicola & 15,0 & 1,8 & 0,2 & 0,6 & 0,4 \\
\hline Waltheria indica & 15,0 & 1,8 & 0,2 & 0,6 & 0,4 \\
\hline Desmodium incanum & 2,5 & 1,8 & 0,0 & 0,5 & 0,3 \\
\hline plântula & 2,5 & 1,8 & 0,0 & 0,5 & 0,3 \\
\hline plântula de Poaceae 1 & 2,5 & 1,8 & 0,0 & 0,5 & 0,3 \\
\hline plântula de Poaceae 2 & 2,5 & 1,8 & 0,0 & 0,5 & 0,3 \\
\hline Solanum viarum & 2,5 & 1,8 & 0,0 & 0,5 & 0,3 \\
\hline Total & $6.345,0$ & 325,0 & 100,0 & 100,0 & 100,0 \\
\hline agosto/2009 & $\begin{array}{l}\text { CA } \\
(\%)\end{array}$ & $\begin{array}{l}\text { FA } \\
(\%)\end{array}$ & $\begin{array}{l}\text { CR } \\
(\%)\end{array}$ & $\begin{array}{l}\text { FR } \\
(\%)\end{array}$ & $\begin{array}{l}\text { VI } \\
(\%)\end{array}$ \\
\hline solo descoberto & $4.263,0$ & 98,2 & 62,5 & 28,3 & 45,4 \\
\hline Acanthospermum australe & 812,5 & 66,1 & 11,9 & 19,1 & 15,5 \\
\hline material morto & 462,5 & 58,9 & 6,9 & 17,1 & 12,0 \\
\hline mantilho & 367,5 & 58,9 & 5,4 & 17,0 & 11,2 \\
\hline Paspalum notatum & 462,5 & 30,3 & 6,9 & 8,7 & 7,8 \\
\hline Urochloa brizantha & 205,0 & 7,1 & 3,0 & 2,0 & 2,5 \\
\hline Urochloa humidicola & 55,0 & 5,4 & 0,8 & 1,6 & 1,2 \\
\hline Mimosa polycarpa & 77,5 & 3,5 & 1,1 & 1,0 & 1,0 \\
\hline Digitaria bicornis & 37,5 & 1,8 & 0,5 & 0,4 & 0,5 \\
\hline Arachis oteroi & 15,0 & 1,8 & 0,2 & 0,6 & 0,4 \\
\hline Sida linifolia & 15,0 & 1,8 & 0,2 & 0,6 & 0,4 \\
\hline Sida rhombifolia & 15,0 & 1,8 & 0,2 & 0,6 & 0,4 \\
\hline Solanum viarum & 15,0 & 1,8 & 0,2 & 0,6 & 0,4 \\
\hline Waltheria indica & 15,0 & 1,8 & 0,2 & 0,6 & 0,4 \\
\hline plântula & 2,5 & 1,8 & 0,0 & 0,6 & 0,3 \\
\hline plântula de Poaceae 1 & 2,5 & 1,8 & 0,0 & 0,6 & 0,3 \\
\hline plântula de Poaceae 2 & 2,5 & 1,8 & 0,0 & 0,6 & 0,3 \\
\hline Total & $6.825,0$ & 344,6 & 100,0 & 100,0 & 100,0 \\
\hline novembro/2009 & $\begin{array}{l}\text { CA } \\
(\%)\end{array}$ & $\begin{array}{l}\text { FA } \\
(\%)\end{array}$ & $\begin{array}{l}\text { CR } \\
(\%)\end{array}$ & $\begin{array}{l}\text { FR } \\
(\%)\end{array}$ & $\begin{array}{c}\text { VI } \\
(\%)\end{array}$ \\
\hline solo descoberto & $3.580,0$ & 98,2 & 50,3 & 26,8 & 38,6 \\
\hline Acanthospermum australe & $1.113,0$ & 69,6 & 15,6 & 19,0 & 17,3 \\
\hline material morto & 635,0 & 62,5 & 8,9 & 17,1 & 13,0 \\
\hline mantilho & 377,5 & 50,0 & 5,4 & 13,6 & $\begin{array}{c}9,5 \\
\text { continuc }\end{array}$ \\
\hline
\end{tabular}


Tabela 4 (continuação)

\begin{tabular}{|c|c|c|c|c|c|}
\hline novembro/2009 & $\begin{array}{l}\text { CA } \\
(\%)\end{array}$ & $\begin{array}{l}\text { FA } \\
(\%)\end{array}$ & $\begin{array}{l}\text { CR } \\
(\%)\end{array}$ & $\begin{array}{l}\text { FR } \\
(\%)\end{array}$ & $\begin{array}{c}\text { VI } \\
(\%)\end{array}$ \\
\hline Paspalum notatum & 590,0 & 30,4 & 8,3 & 8,3 & 8,3 \\
\hline Urochloa brizantha & 350,0 & 12,5 & 4,9 & 3,4 & 4,2 \\
\hline Urochloa humidicola & 147,5 & 10,7 & 2,1 & 2,9 & 2,5 \\
\hline Mimosa polycarpa & 100,0 & 3,6 & 1,4 & 1,0 & 1,2 \\
\hline Digitaria bicornis & 77,5 & 3,6 & 1,1 & 1,0 & 1,0 \\
\hline Sida rhombifolia & 32,5 & 5,3 & 0,5 & 1,5 & 1,0 \\
\hline plântula de $A$. australe & 5,0 & 3,5 & 0,2 & 1,0 & 0,5 \\
\hline Arachis oteroi & 37,5 & 1,8 & 0,5 & 0,5 & 0,5 \\
\hline Senna occidentalis & 15,0 & 1,8 & 0,2 & 0,5 & 0,4 \\
\hline Sida linifolia & 15 & 1,8 & 0,2 & 0,5 & 0,4 \\
\hline Solanum viarum & 15 & 1,8 & 0,2 & 0,5 & 0,4 \\
\hline Waltheria indica & 15 & 1,8 & 0,2 & 0,5 & 0,4 \\
\hline Crotalaria incana & 2,5 & 1,8 & 0,0 & 0,5 & 0,2 \\
\hline plântula de Malvaceae & 2,5 & 1,8 & 0,0 & 0,5 & 0,2 \\
\hline plântula de Rubiaceae & 2,5 & 1,8 & 0,0 & 0,5 & 0,2 \\
\hline plântula de $U$. brizantha & 2,5 & 1,8 & 0,0 & 0,4 & 0,2 \\
\hline Total & $7.115,0$ & 366,1 & 100,0 & 100,0 & 100,0 \\
\hline fevereiro/2010 & $\begin{array}{l}\text { CA } \\
(\%)\end{array}$ & $\begin{array}{l}\text { FA } \\
(\%)\end{array}$ & $\begin{array}{l}\text { CR } \\
(\%)\end{array}$ & $\begin{array}{l}\text { FR } \\
(\%)\end{array}$ & $\begin{array}{l}\text { VI } \\
(\%)\end{array}$ \\
\hline solo descoberto & $3.562,5$ & 100,0 & 45,3 & 23,1 & 34,2 \\
\hline Acanthospermum australe & 977,5 & 73,2 & 12,5 & 16,9 & 14,7 \\
\hline material morto & 737,5 & 73,2 & 9,4 & 16,9 & 13,2 \\
\hline mantilho & 602,5 & 66,1 & 7,7 & 15,3 & 11,5 \\
\hline Paspalum notatum & 672,5 & 35,7 & 8,6 & 8,3 & 8,4 \\
\hline Urochloa brizantha & 510 & 23,2 & 6,5 & 5,4 & 5,9 \\
\hline Urochloa humidicola & 325 & 19,6 & 4,1 & 4,6 & 4,3 \\
\hline Digitaria bicornis & 105 & 7,1 & 1,3 & 1,7 & 1,5 \\
\hline Mimosa polycarpa & 115 & 5,3 & 1,5 & 1,2 & 1,4 \\
\hline Sida rhombifolia & 35 & 7,1 & 0,5 & 1,7 & 1,1 \\
\hline Senna occidentalis & 62,5 & 1,8 & 0,7 & 0,5 & 0,6 \\
\hline Arachis oteroi & 15,0 & 1,8 & 0,2 & 0,4 & 0,3 \\
\hline Chamaecrista serpens & 15,0 & 1,8 & 0,2 & 0,4 & 0,3 \\
\hline Corchorus hirtus & 15,0 & 1,8 & 0,2 & 0,4 & 0,3 \\
\hline Desmodium incanum & 15,0 & 1,8 & 0,2 & 0,4 & 0,3 \\
\hline Ipomoea grandifolia & 15,0 & 1,8 & 0,2 & 0,4 & 0,3 \\
\hline Paspalum plicatulum & 15,0 & 1,8 & 0,2 & 0,4 & 0,3 \\
\hline Setaria parviflora & 15,0 & 1,8 & 0,2 & 0,4 & 0,3 \\
\hline Sida linifolia & 15,0 & 1,8 & 0,2 & 0,4 & 0,3 \\
\hline Solanum paniculatum & 15,0 & 1,8 & 0,2 & 0,4 & 0,3 \\
\hline Solanum viarum & 15,0 & 1,8 & 0,2 & 0,4 & 0,3 \\
\hline Senna obtusifolia & 2,5 & 1,8 & 0,0 & 0,4 & 0,2 \\
\hline Total & $7.857,5$ & 432,1 & 100,0 & 100,0 & 100,0 \\
\hline
\end{tabular}

$72,8 \%$ a $47,1 \%$ na encosta da voçoroca (tabelas 5 , 6). "Material morto" e "mantilho" somaram ao longo do ano estudado VI de $18,0 \%$ a $25,0 \%$ no centro e de $20,4 \%$ a $25,9 \%$ na encosta da voçoroca.

Acanthospermum australe, dentre as espécies observadas, obteve os mais altos valores de VI no centro $(9,1 \%$ a $15,7 \%)$ e na encosta $(13,7 \%$ a $14,7 \%)$. As demais espécies (CR de 10,9\% a 29,5\% no centro e de $8,2 \%$ a $22,8 \%$ na encosta) pouco contribuíram com a cobertura vegetal e portanto com o processo de revegetação durante o período de estudo.

A inclinação e instabilidade das encostas de voçorocas podem dificultar a fixação da maioria das espécies, comprometendo assim a revegetação, entretanto, observou-se um aumento considerável na cobertura vegetal e início de deposição de sedimentos 
Tabela 5. Dados fitossociológicos de atributos e espécies em quatro épocas, no centro de uma voçoroca em pastagem cultivada com Urochloa brizantha após exclusão do gado, Fazenda Nova Esperança, Sidrolândia, MS, Brasil. VI: Valor de Importância; CA: Cobertura Absoluta; FA: Frequência Absoluta; CR: Cobertura Relativa; FR: Frequência Relativa.

Table 5. Phytosociological data of attributes and species in four seasons in the center of a gully in cultivated pasture with Urochloa brizantha after cattle exclusion, Fazenda Nova Esperança, Sidrolândia, MS, Brazil. VI: Importance Value; CA: Absolute Coverage; FA: Absolute Frequency, CR: Relative Coverage; FR: Relative Frequency.

\begin{tabular}{|c|c|c|c|c|c|}
\hline maio/2009 & $\begin{array}{l}\text { CA } \\
(\%)\end{array}$ & $\begin{array}{l}\text { FA } \\
(\%)\end{array}$ & $\begin{array}{l}\text { CR } \\
(\%)\end{array}$ & $\begin{array}{l}\text { FR } \\
(\%)\end{array}$ & $\begin{array}{c}\text { VI } \\
(\%)\end{array}$ \\
\hline solo descoberto & $1.590,0$ & 33,9 & 75,4 & 37,3 & 56,4 \\
\hline material morto & 130,0 & 14,3 & 6,2 & 15,6 & 10,9 \\
\hline Acanthospermum australe & 92,5 & 12,5 & 4,4 & 13,7 & 9,1 \\
\hline mantilho & 65,0 & 10,7 & 3,1 & 11,7 & 7,4 \\
\hline Paspalum notatum & 67,5 & 5,4 & 3,2 & 5,8 & 4,5 \\
\hline Urochloa brizantha & 85,0 & 1,8 & 4,1 & 2,0 & 3,0 \\
\hline Mimosa polycarpa & 40,0 & 3,7 & 1,9 & 3,9 & 2,9 \\
\hline Arachis oteroi & 15,0 & 1,8 & 0,7 & 2,0 & 1,4 \\
\hline Digitaria bicornis & 15,0 & 1,8 & 0,7 & 2,0 & 1,4 \\
\hline plântula & 2,5 & 1,7 & 0,1 & 2,0 & 1,0 \\
\hline plântula de Poaceae 1 & 2,5 & 1,7 & 0,1 & 2,0 & 1,0 \\
\hline plântula de Poaceae 2 & 2,5 & 1,7 & 0,1 & 2,0 & 1,0 \\
\hline Total & $2.107,5$ & 91,0 & 100,0 & 100,0 & 100,0 \\
\hline agosto/2009 & $\begin{array}{l}\text { CA } \\
(\%)\end{array}$ & $\begin{array}{l}\text { FA } \\
(\%)\end{array}$ & $\begin{array}{l}\text { CR } \\
(\%)\end{array}$ & $\begin{array}{l}\text { FR } \\
(\%)\end{array}$ & $\begin{array}{c}\text { VI } \\
(\%)\end{array}$ \\
\hline solo descoberto & $1.420,0$ & 94,7 & 63,0 & 29,5 & 46,3 \\
\hline Acanthospermum australe & 197,5 & 63,2 & 8,8 & 19,7 & 14,2 \\
\hline mantilho & 150,0 & 57,8 & 6,7 & 18,0 & 12,4 \\
\hline material morto & 120,0 & 42,1 & 5,3 & 13,1 & 9,2 \\
\hline Paspalum notatum & 130,0 & 21,1 & 5,8 & 6,7 & 6,2 \\
\hline Mimosa polycarpa & 77,5 & 10,4 & 3,4 & 3,4 & 3,4 \\
\hline Urochloa brizantha & 97,5 & 5,3 & 4,3 & 1,6 & 3,0 \\
\hline Digitaria bicornis & 37,5 & 5,3 & 1,7 & 1,6 & 1,7 \\
\hline Arachis oteroi & 15,0 & 5,2 & 0,7 & 1,6 & 1,2 \\
\hline plântula & 2,5 & 5,3 & 0,1 & 1,6 & 0,8 \\
\hline plântula de Poaceae 1 & 2,5 & 5,3 & 0,1 & 1,6 & 0,8 \\
\hline plântula de Poaceae 2 & 2,5 & 5,3 & 0,1 & 1,6 & 0,8 \\
\hline Total & $2.252,5$ & 321,0 & 100,0 & 100,0 & 100,0 \\
\hline novembro/2009 & $\begin{array}{l}\text { CA } \\
(\%)\end{array}$ & $\begin{array}{l}\text { FA } \\
(\%)\end{array}$ & $\begin{array}{l}\text { CR } \\
(\%)\end{array}$ & $\begin{array}{l}\text { FR } \\
(\%)\end{array}$ & $\begin{array}{c}\text { VI } \\
(\%)\end{array}$ \\
\hline solo descoberto & $1.172,5$ & 94,7 & 48,6 & 27,3 & 37,9 \\
\hline Acanthospermum australe & 327,5 & 68,4 & 13,6 & 19,7 & 16,6 \\
\hline material morto & 192,5 & 47,4 & 8,0 & 13,6 & 10,8 \\
\hline mantilho & 147,5 & 52,6 & 6,1 & 15,2 & 10,7 \\
\hline Paspalum notatum & 210,0 & 21,1 & 8,7 & 6,1 & 7,4 \\
\hline Urochloa brizantha & 127,5 & 15,8 & 5,3 & 4,6 & 4,9 \\
\hline Mimosa polycarpa & 100,0 & 10,5 & 4,1 & 3,0 & 3,6 \\
\hline Digitaria bicornis & 77,5 & 10,4 & 3,1 & 3,0 & 3,1 \\
\hline Arachis oteroi & 37,5 & 5,3 & 1,6 & 1,5 & 1,5 \\
\hline Senna occidentalis & 15,0 & 5,3 & 0,6 & 1,5 & 1,1 \\
\hline Crotalaria incana & 2,5 & 5,3 & 0,1 & 1,5 & 0,8 \\
\hline plântula de Rubiaceae & 2,5 & 5,3 & 0,1 & 1,5 & 0,8 \\
\hline Sida rhombifolia & 2,5 & 5,3 & 0,1 & 1,5 & 0,8 \\
\hline Total & $2.415,0$ & 347,4 & 100,0 & 100,0 & 100,0 \\
\hline
\end{tabular}


Tabela 5 (continuação)

\begin{tabular}{|c|c|c|c|c|c|}
\hline fevereiro/2010 & $\begin{array}{l}\text { CA } \\
(\%)\end{array}$ & $\begin{array}{l}\text { FA } \\
(\%)\end{array}$ & $\begin{array}{l}\text { CR } \\
(\%)\end{array}$ & $\begin{array}{l}\text { FR } \\
(\%)\end{array}$ & $\begin{array}{l}\text { VI } \\
(\%)\end{array}$ \\
\hline solo descoberto & $1.152,5$ & 294,7 & 42,0 & 24,1 & 33,1 \\
\hline Acanthospermum australe & 377,5 & 215,8 & 13,8 & 17,7 & 15,7 \\
\hline material morto & 192,5 & 215,8 & 7,0 & 17,7 & 12,3 \\
\hline mantilho & 212,5 & 194,7 & 7,7 & 16,0 & 11,9 \\
\hline Urochloa brizantha & 277,5 & 68,4 & 10,1 & 5,6 & 7,9 \\
\hline Paspalum notatum & 190,0 & 105,3 & 6,9 & 8,6 & 7,8 \\
\hline Urochloa humidicola & 30,0 & 57,9 & 1,1 & 4,7 & 2,9 \\
\hline Digitaria bicornis & 105,0 & 21,1 & 3,8 & 1,7 & 2,8 \\
\hline Mimosa polycarpa & 115,0 & 15,7 & 4,2 & 1,3 & 2,7 \\
\hline Senna occidentalis & 62,5 & 5,2 & 2,3 & 0,5 & 1,3 \\
\hline Sida rhombifolia & 15,0 & 21,1 & 0,6 & 1,7 & 1,1 \\
\hline Arachis oteroi & 15,0 & 5,3 & 0,6 & 0,4 & 0,5 \\
\hline Total & $2.745,0$ & $1.221,0$ & 100,0 & 100,0 & 100,0 \\
\hline
\end{tabular}

Tabela 6. Dados fitossociológicos de atributos e espécies em quatro épocas, nas encostas de uma voçoroca em pastagem cultivada com Urochloa brizantha após exclusão do gado, Fazenda Nova Esperança, Sidrolândia, MS, Brasil. VI: Valor de Importância; CA: Cobertura Absoluta; FA: Frequência Absoluta; CR: Cobertura Relativa; FR: Frequência Relativa.

Table 6. Phytosociological data of attributes and species in four seasons in the slopes of a gully in cultivated pasture with Urochloa brizantha after cattle exclusion, Fazenda Nova Esperança, Sidrolândia, MS, Brazil. VI: Importance Value; CA: Absolute Coverage; FA: Absolute Frequency, CR: Relative Coverage; FR: Relative Frequency.

\begin{tabular}{|c|c|c|c|c|c|}
\hline maio/2009 & $\begin{array}{l}\text { CA } \\
(\%)\end{array}$ & $\begin{array}{l}\text { FA } \\
(\%)\end{array}$ & $\begin{array}{l}\text { CR } \\
(\%)\end{array}$ & $\begin{array}{l}\text { FR } \\
(\%)\end{array}$ & $\begin{array}{l}\text { VI } \\
(\%)\end{array}$ \\
\hline solo descoberto & $3.045,0$ & 100,0 & 72,9 & 28,9 & 50,9 \\
\hline Acanthospermum australe & 430,0 & 59,5 & 10,3 & 17,2 & 13,7 \\
\hline material morto & 240,0 & 59,5 & 5,7 & 17,2 & 11,5 \\
\hline mantilho & 122,5 & 51,4 & 2,9 & 14,8 & 8,9 \\
\hline Paspalum notatum & 167,5 & 35,1 & 4,0 & 10,3 & 7,1 \\
\hline Sida rhombifolia & 65,0 & 16,2 & 1,5 & 4,6 & 3,1 \\
\hline Urochloa brizantha & 60,0 & 10,8 & 1,3 & 3,0 & 2,2 \\
\hline Sida linifolia & 15,0 & 2,7 & 0,4 & 0,8 & 0,6 \\
\hline Urochloa humidicola & 15,0 & 2,7 & 0,4 & 0,8 & 0,6 \\
\hline Waltheria indica & 15,0 & 2,7 & 0,4 & 0,8 & 0,6 \\
\hline Desmodium incanum & 2,5 & 2,7 & 0,1 & 0,8 & 0,4 \\
\hline Solanum viarum & 2,5 & 2,7 & 0,1 & 0,8 & 0,4 \\
\hline Total & $4.180,0$ & 346,0 & 100,0 & 100,0 & 100,0 \\
\hline agosto/2009 & $\begin{array}{l}\text { CA } \\
(\%)\end{array}$ & $\begin{array}{l}\text { FA } \\
(\%)\end{array}$ & $\begin{array}{l}\text { CR } \\
(\%)\end{array}$ & $\begin{array}{l}\text { FR } \\
(\%)\end{array}$ & $\begin{array}{l}\text { VI } \\
(\%)\end{array}$ \\
\hline solo descoberto & $2.842,5$ & 102,7 & 62,2 & 28,2 & 45,2 \\
\hline Acanthospermum australe & 615,0 & 70,3 & 13,5 & 19,3 & 16,4 \\
\hline material morto & 342,5 & 67,6 & 7,5 & 18,6 & 13,0 \\
\hline mantilho & 217,5 & 59,5 & 4,8 & 16,3 & 10,5 \\
\hline Paspalum notatum & 332,5 & 35,1 & 7,3 & 9,6 & 8,5 \\
\hline Urochloa brizantha & 107,5 & 10,8 & 2,3 & 3,0 & 2,7 \\
\hline Urochloa humidicola & 55,0 & 8,1 & 1,2 & 2,2 & 1,7 \\
\hline Sida linifolia & 15,0 & 2,7 & 0,3 & 0,7 & 0,5 \\
\hline Sida rhombifolia & 15,0 & 2,7 & 0,3 & 0,7 & 0,5 \\
\hline Solanum viarum & 15,0 & 2,7 & 0,3 & 0,7 & 0,5 \\
\hline Waltheria indica & 15,0 & 2,7 & 0,3 & 0,7 & 0,5 \\
\hline Total & $4.572,5$ & 364,9 & 100,0 & 100,0 & $\begin{array}{c}100,0 \\
\text { continuo }\end{array}$ \\
\hline
\end{tabular}


Tabela 6 (continuação)

\begin{tabular}{|c|c|c|c|c|c|}
\hline novembro/2009 & $\begin{array}{l}\text { CA } \\
(\%)\end{array}$ & $\begin{array}{l}\text { FA } \\
(\%)\end{array}$ & $\begin{array}{l}\text { CR } \\
(\%)\end{array}$ & $\begin{array}{l}\text { FR } \\
(\%)\end{array}$ & $\begin{array}{l}\text { VI } \\
(\%)\end{array}$ \\
\hline solo descoberto & $2.407,5$ & 100,0 & 51,3 & 26,6 & 39,0 \\
\hline Acanthospermum australe & 785,0 & 75,7 & 16,7 & 20,1 & 18,4 \\
\hline material morto & 442,5 & 70,3 & 9,4 & 18,7 & 14,1 \\
\hline mantilho & 230,0 & 48,7 & 4,9 & 13,1 & 9,0 \\
\hline Paspalum notatum & 380,0 & 35,1 & 8,1 & 9,3 & 8,7 \\
\hline Urochloa brizantha & 222,5 & 10,8 & 4,7 & 2,9 & 3,8 \\
\hline Urochloa humidicola & 147,5 & 16,2 & 3,1 & 4,3 & 3,7 \\
\hline Sida rhombifolia & 30,0 & 5,4 & 0,7 & 1,5 & 1,0 \\
\hline Sida linifolia & 15,0 & 2,7 & 0,3 & 0,7 & 0,5 \\
\hline Solanum viarum & 15,0 & 2,7 & 0,3 & 0,7 & 0,5 \\
\hline Waltheria indica & 15,0 & 2,7 & 0,3 & 0,7 & 0,5 \\
\hline plântula de Malvaceae & 2,5 & 2,7 & 0,1 & 0,7 & 0,4 \\
\hline plântula de $U$. brizantha & 2,5 & 2,7 & 0,1 & 0,7 & 0,4 \\
\hline Total & $4.695,0$ & 375,7 & 100,0 & 100,0 & 100,0 \\
\hline fevereiro/2010 & $\begin{array}{l}\text { CA } \\
(\%)\end{array}$ & $\begin{array}{l}\text { FA } \\
(\%)\end{array}$ & $\begin{array}{l}\text { CR } \\
(\%)\end{array}$ & $\begin{array}{l}\text { FR } \\
(\%)\end{array}$ & $\begin{array}{l}\text { VI } \\
(\%)\end{array}$ \\
\hline solo descoberto & $2.410,0$ & 151,4 & 47,1 & 24,0 & 35,6 \\
\hline Acanthospermum australe & 600,0 & 110,8 & 11,7 & 17,6 & 14,6 \\
\hline material morto & 45,0 & 110,8 & 10,6 & 17,6 & 14,0 \\
\hline mantilho & 390,0 & 100,0 & 7,6 & 15,9 & 11,7 \\
\hline Paspalum notatum & 482,5 & 54,1 & 9,4 & 8,6 & 9,0 \\
\hline Urochloa humidicola & 295,0 & 29,7 & 5,8 & 4,8 & 5,2 \\
\hline Urochloa brizantha & 232,5 & 35,1 & 4,6 & 5,7 & 5,0 \\
\hline Sidarhombifolia & 20,0 & 10,8 & 0,4 & 1,8 & 1,1 \\
\hline Chamaecrista serpens & 15,0 & 2,7 & 0,3 & 0,4 & 0,4 \\
\hline Corchorus hirtus & 15,0 & 2,7 & 0,3 & 0,4 & 0,4 \\
\hline Desmodium incanum & 15,0 & 2,7 & 0,3 & 0,4 & 0,4 \\
\hline Ipomoea grandifolia & 15,0 & 2,7 & 0,3 & 0,4 & 0,4 \\
\hline Paspalum plicatulum & 15,0 & 2,7 & 0,3 & 0,4 & 0,4 \\
\hline Setaria parviflora & 15,0 & 2,7 & 0,3 & 0,4 & 0,4 \\
\hline Sida linifolia & 15,0 & 2,7 & 0,3 & 0,4 & 0,4 \\
\hline Solanum paniculatum & 15,0 & 2,7 & 0,3 & 0,4 & 0,4 \\
\hline Solanum viarum & 15,0 & 2,7 & 0,3 & 0,4 & 0,4 \\
\hline Senna obtusifolia & 2,5 & 2,7 & 0,1 & 0,4 & 0,2 \\
\hline Total & $5.112,5$ & 629,7 & 100,0 & 100,0 & 100,0 \\
\hline
\end{tabular}

no fundo, de até $50 \mathrm{~cm}$ de espessura. Inicialmente, formada por gramíneas estoloníferas, algumas exóticas como Urochloa humidicola, e outras poucas ervas, a cobertura das encostas foi sendo gradualmente enriquecida por espécies nativas com diferentes formas de vida, crescimento e hábitos, inclusive o arbustivo, como Solanum paniculatum (tabela 6), merecendo destaque também a capacidade de Stylosanthes spp. de vegetar nesse subsolo exposto e ressecado.

Conclui-se que a revegetação natural na voçoroca estudada está em curso, sendo favorecida pela exclusão do gado e caracterizada pela ocorrência de: considerável número de espécies pertencentes a diferentes famílias de fanerógamas; grupos de espécies com diferentes hábitos, formas de vida e de crescimento; produção de flores e frutos ao longo de todo o ano; espécies com diferentes formas de dispersão; pioneiras importantes como as leguminosas; espécies utilizadas na recuperação de áreas degradadas; e competidoras agressivas como Urochloa spp. Sugere-se que o estudo seja repetido daqui a alguns anos, para monitorar a sucessão da vegetação. 


\section{Agradecimentos}

Ao Fernando Augusto Barcelos de Brum e à Cristina Saliés, proprietários da Fazenda Nova Esperança. A primeira autora agradece à CAPES pela bolsa Prodoc concedida entre 2006 e 2009 e por parte do recurso financeiro utilizado na execução do trabalho. O terceiro autor agradece a bolsa Produtividade Científica 2 do CNPq.

\section{Literatura citada}

AGRAER(Agência de Desenvolvimento Agrário e Extensão Rural). 2010. Boletins meteorológicos. http:// www.agraer.ms.gov.br/cemtec (acesso em 27.8.2010).

Andrade, A.G., Portocarrero, H. \& Capeche, C.L. 2005. Práticas mecânicas e vegetativas para controle de voçorocas. EMBRAPA. Comunicado Técnico 33: 1-4.

Andres, J. \& Werlang, M.K. 2006. A influência do uso da terra nos processos erosivos: correlação entre os índices de concentração de nascentes (ICN) e de voçorocas (ICV) no oeste do município de Pirapó, RS. Ciência e Natura 28: 35-44.

Antunes, N.B. \& Ribeiro, J.F. 1999. Aspectos fenológicos de seis espécies vegetais em matas de galeria do Distrito Federal. Pesquisa Agropecuária Brasileira 34: 1517-1527.

APG(Angiosperm Phylogeny Group) III. 2009. An update of the Angiosperm Phylogeny Group classification for the orders and families of flowering plants: APG III. Botanical Journal of Linnean Society 161: 105-121.

Bahia, V.G., Curi, N., Carmo, D.N. \& Marques, J.J.G.S.M. 1992. Fundamentos de erosão do solo: tipos, formas, mecanismos, fatores determinantes e controle. Informe Agropecuário 16: 25-31.

Batalha, M.A., Aragaki, S. \& Mantovani, W. 1997. Variações fenológicas das espécies do Cerrado em Emas (Pirassununga, SP). Acta Botanica Brasilica 11: 61-78.

Batalha, M.A. \& Mantovani, W. 2000. Reproductive phenological patterns of Cerrado plant species at the Pé-de-Gigante Reserve (Santa Rita do Passa Quatro, SP, Brazil): a comparasion between the herbaceous and woody floras. Revista Brasileira Biologia 60: 129-145.

Braun-Blanquet, J. 1979. Fitosociologia: bases para el estudio de las comunidades vegetales. H. Blume Ediciones, Madrid.

Comissão de Fertilidade do Solo - RS/SC. 1995. Recomendações de adubação e de calagem para os estados do Rio Grande do Sul e de Santa Catarina. 3 ed. SBCS - Núcleo Regional Sul, Passo Fundo.

Comissão de Fertilidade do Solo - RS/SC. 2004. Recomendações de adubação e de calagem para os estados do Rio Grande do Sul e de Santa Catarina. 10 ed. SBCS - Núcleo Regional Sul, Passo Fundo.
Embrapa Solos. 2008. Sistema Brasileiro de Classificação de Solos. http://www.cnps.embrapa.br/sibcs (acesso em 1.9.2008).

Gandolfi, S. \& Rodrigues, R.R. 2007. Metodologias de restauração florestal. In: R. Mazzuchelli (coord.). Manejo ambiental e restauração de áreas degradadas. Cargill, São Paulo, pp. 109-144.

Guerra, A.J.T. 2001. Processos erosivos nas encostas. In: A.J.T. Guerra \& S.D. Cunha (orgs.). Geomorfologia: uma atualização de bases e conceitos. 4 ed. Bertrand Brasil, Rio de Janeiro, pp. 149-209.

Junk, W.J. \& Piedade, M.T.F. 1997. Plant life in the floodplain with special reference to herbaceous plants. In: W.J. Junk (ed.). The Central Amazon floodplain: Ecology of a pulsing system. Springer-Verlag, Berlin, pp. 147-185.

Hammer, O., Harper, D.A.T. \& Ryan, P.D. 2001. PAST: Palaeontological Statistics software package for education and data analysis. Palaeontologia Electronica, 4: 1-9.

Kissmann, K. 1997. Plantas infestantes e nocivas. Tomo I. 2 ed. BASF, São Paulo.

Kissmann, K.G. \& Groth, D. 1999. Plantas infestantes e nocivas. Tomo II. 2 ed. BASF, São Paulo.

Leite, L.L., Martins, C.R. \& Haridasan, M. 1994. Efeito da descompactação e adubação do solo na revegetação espontânea de uma cascalheira no Parque Nacional de Brasília. In: M. Balensiefer, A.J. Araújo \& N.C. Rosot (eds.). Anais do I Simpósio Sul-Americano e II Simpósio Nacional sobre Recuperação de Áreas Degradadas, Foz do Iguaçu, pp. 527-534.

Lemos, R.C. \& Santos, R.D. 1996. Manual de descrição e coleta de solo no campo. 3 ed. Sociedade Brasileira de Ciência do Solo, Campinas.

Lorenzi, H. 2000. Plantas daninhas do Brasil: terrestres, aquáticas, parasitas e tóxicas. 3 ed. Instituto Plantarum, Nova Odessa.

Macedo, J.R., Capeche, C.L. \& Macedo, A.S. 2009. Recomendação de manejo e conservação de solo e água. Programa Rio Rural Manual Técnico 20: 1-45.

Mantovani, W. \& Martins, F.R. 1988. Variações fenológicas das espécies do cerrado da Reserva Biológica de MojiGuaçu, Estado de São Paulo. Revista. Brasileira de Botânica 11: 101-112.

Matteucci, S.D. \& Colma, A. 1982. Metodologia para el estudio de la vegetación. Secretaria General de la Organización de los Estados Americanos, Washington.

Morretes, B.L. 1992. Potencialidades e restrições da regeneração natural na recuperação de áreas degradadas. In: M. Balensiefer (ed.). Anais do Simpósio Nacional de Recuperação de Áreas Degradadas, Curitiba, pp. 8-16. 
Müller-Dombois, D. \& Ellenberg, H. 1974. Aims and methods of vegetation ecology. John Wiley \& Sons, New York.

Oliveira, D.E.C., Silva,A.V.,Almeida,A.F.,Sai, E.F.\& Raymundo Junior, O. 2010. Fungos micorrízicos arbusculares e rizóbio no crescimento inicial de Acacia mangium Willd. em solo de mineração da região sudoeste do estado de Goiás. Global Science and Technology 3: 1-10.

Pedreira, C.G.S. \& Pedreira, B.C. 2006. A grama-forquilha (Paspalum notatum). In: M. Dall'agnol, C. Nabinger, L.M. Rosa, J.L.S. Silva, D.T. Santos \& R.J. Santos (eds.). Anais do I Simpósio de Forrageiras e Produção Animal, Porto Alegre, pp. 115-146.

Radford, A.E., Dickison, W.C., Massey, J.R. \& Bell, C.R. 1974. Vascular plant systematics. Harper \& Row, New York.

Rocha, E.A.V., Alves, R.R., Bezerra, J.F.R. \& Rodrigues, S.C. 2005. Monitoramento de voçoroca no município de Ipameri - GO. In: II Simpósio Sobre Solos Tropicais e Processos Erosivos no Centro-Oeste, Goiânia, pp. 59-64.

Regensburger, B. 2004. Recuperação de áreas degradadas pela mineração de argila através da regularização topográfica, da adição de insumos e serrapilheira e de atratores da fauna. Dissertação de Mestrado em Agroecossistema, Universidade Federal de Santa Catarina, Florianópolis.
Seitz, R.A. 1994. A regeneração natural na recuperação de áreas degradadas. In: M. Balensiefer, A.J. Araújo \& N.C. Rosot (eds.). Anais do I Simpósio Sulamericano e II Simpósio Nacional de Recuperação de Áreas Degradadas, Foz do Iguaçu, pp. 103-110.

Tomé Júnior, J.B. 1997. Manual para interpretação de análise de solo. Agropecuária, Guaíba.

Silva, F.C., Eira, P.A., Barreto, W.O., Perez, D.V. \& Silva, C.A. 1998. Análises químicas para avaliação da fertilidade do solo. Métodos usados na Embrapa Solos. Embrapa Solos Documentos 3: 1-40.

Soil Science Society of America. 2010. Glossary of Soil Science Terms. https://soils.org/publications/soilsglossary. (acesso em 10.5.2010).

Souza Filho, A.P.S., Rodrigues, L.R.A. \& Rodrigues, T.J.D. 1997. Potencial alelopático de forrageiras tropicais: efeitos sobre invasoras de pastagens. Planta Daninha 15: 54-60.

Van der Pijl, L. 1982. Principles of dispersal in higher plants. Springer-Verlag, New York.

Weyser, V.L. \& Godoy, S.A.P. 2001. Florística de um hectare de cerrado stricto sensu na ARIE - Cerrado Pé-deGigante, Santa Rita do Passa Quatro, SP. Acta Botanica Brasilica 15:201-212. 